Steady boundary-layer solutions for a swirling stratified fluid in a rotating cone

Hewitt, R.E. and Duck, P.W. and Foster, M.R.

1999

MIMS EPrint: 2013.66

Manchester Institute for Mathematical Sciences

School of Mathematics

The University of Manchester

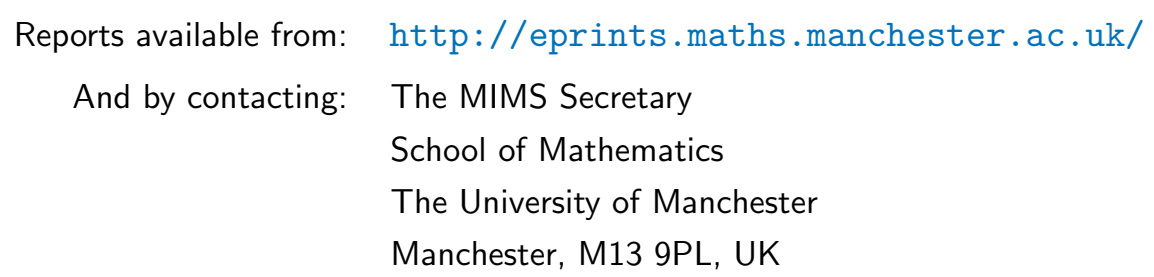

ISSN 1749-9097 


\title{
Steady boundary-layer solutions for a swirling stratified fluid in a rotating cone
}

\author{
By R. E. HEWITT ${ }^{1}$, P. W. DUCK ${ }^{1}$ AND M. R. FOSTER ${ }^{2}$ \\ ${ }^{1}$ Department of Mathematics, University of Manchester, \\ Oxford Road, Manchester, M13 9PL, UK \\ ${ }^{2}$ Department of Aerospace Engineering, Applied Mechanics and Aviation, \\ The Ohio State University, Columbus, Ohio, 43210, USA \\ (Received 11 May 1998 and in revised form 26 October 1998)
}

We consider a set of nonlinear boundary-layer equations that have been derived by Duck, Foster \& Hewitt (1997a, DFH), for the swirling flow of a linearly stratified fluid in a conical container. In contrast to the unsteady analysis of DFH, we restrict attention to steady solutions and extend the previous discussion further by allowing the container to both co-rotate and counter-rotate relative to the contained swirling fluid. The system is governed by three parameters, which are essentially nondimensional measures of the rotation, stratification and a Schmidt number. Some of the properties of this system are related (in some cases rather subtly) to those found in the swirling flow of a homogeneous fluid above an infinite rotating disk; however, the introduction of buoyancy effects with a sloping boundary leads to other (new) behaviours. A general description of the steady solutions to this system proves to be rather complicated and shows many interesting features, including non-uniqueness, singular solutions and bifurcation phenomena.

We present a broad description of the steady states with particular emphasis on boundaries in parameter space beyond which steady states cannot be continued.

A natural extension of this work (motivated by recent experimental results) is to investigate the possibility of solution branches corresponding to non-axisymmetric boundary-layer states appearing as bifurcations of the axisymmetric solutions. In an Appendix we give details of an exact, non-axisymmetric solution to the NavierStokes equations (with axisymmetric boundary conditions) corresponding to the flow of homogeneous fluid above a rotating disk.

\section{Introduction and formulation}

The recent work of Duck, Foster \& Hewitt (1997a, hereafter referred to as DFH), MacCready \& Rhines (1991) and Hewitt, Davies, Duck \& Foster (1999, hereafter referred to as HDDF), has considered the influence of buoyancy in boundary layers that are commonly found in spin-up problems. The work of DFH considered the boundary layer arising in the spin-up of a rotating stratified fluid in a container with sloping walls (a cone being the chosen geometry). In the analysis of DFH and the following papers (Hewitt et al. 1997b; HDDF), it was noted that a description of the steady states available for the governing boundary-layer equations and an analysis of their stability is a non-trivial exercise. Although a stability analysis was given for some of the available steady states by HDDF, a complete analysis of all the features of the problem is still lacking. In particular, as a first step towards the eventual development 

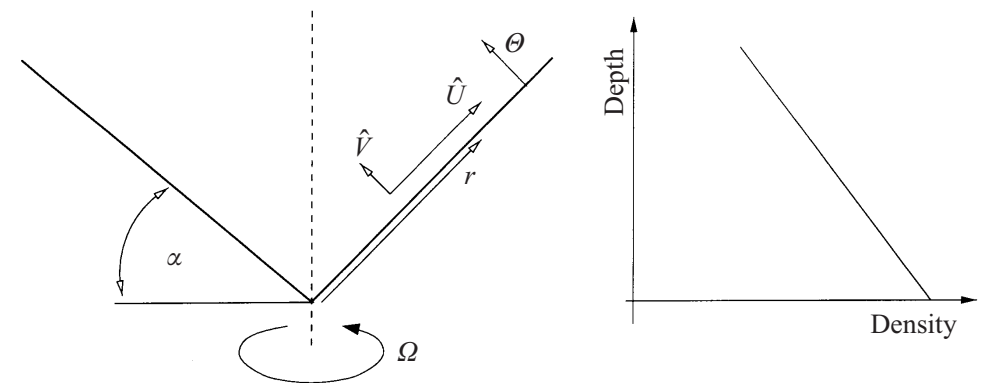

FiguRE 1. A schematic of the geometry and coordinate system. $\Theta$ is a scaled boundary-layer coordinate. $\hat{U}(\Theta), \hat{V}(\Theta)$ are radial and normal velocity components in the boundary layer, and are introduced by a similarity-type solution of von Kármán form, which removes the radial dependence. The fluid density in the interior flow is a linear function of the fluid depth. The cone-angle $\alpha$ can be scaled out of the boundary-layer equations.

of a stability analysis, we note that there has been little discussion concerning nonuniqueness of the steady solution branches, or any investigation of where (in terms of the three-dimensional parameter space) steady solutions exist. Furthermore, in all the analyses of the stratified, spin-up process it has been assumed that the sense of rotation of the fluid at the boundary-layer edge is always the same as the container, that is, there is no counter-rotation.

For a swirling flow of homogeneous fluid above an infinite rotating disk, the effects of counter-rotation have been shown to lead to qualitatively different behaviour in both the steady and unsteady cases. Bodonyi \& Stewartson (1977) (with a later amendment by Stewartson, Simpson \& Bodonyi 1982) examined the unsteady boundary layer that forms adjacent to a rotating disk subsequent to an impulsive change in the sign of the disk's angular frequency. The boundary-layer solution was shown to break down at a finite time, with the displacement thickness becoming infinite. This is a fundamentally different form of breakdown to that identified by DFH for a stratified, sloping, boundary layer. In a later paper the numerical approach utilized by Bodonyi \& Stewartson was extended by Bodonyi (1978) to consider more general counter-rotating (or 'spin-over') problems for rotating disks. Bodonyi presented numerical solutions of the initial value problem, which showed a limit-cycle behaviour.

For the steady-state rotating disk problem, allowing the disk to counter-rotate reveals a fold in the primary branch of solution; we denote the solution arising directly from the classical von Kármán (1921) state as being the primary branch. The solution can be continued beyond this first fold to successively higher branches. We shall discuss this behaviour in more detail later.

The configuration that we wish to consider here is that of a swirling, linearly stratified fluid in a rotating conical container (see figure 1). In particular we take the Ekman number $\left(E=v / \Omega h^{2}\right)$ to be small and discuss the steady boundary layer. Here, $v$ is the kinematic viscosity of the fluid, $\Omega$ is the rotation rate of the cone and $h$ a typical lengthscale. The governing Boussinesq boundary-layer equations for solutions of a similarity type are

$$
\begin{gathered}
\hat{U}^{2}+\hat{V} \hat{U}_{\Theta}-\hat{W}^{2}=\hat{U}_{\Theta \Theta}-B^{*}, \\
2 \hat{U} \hat{W}+\hat{V} \hat{W}_{\Theta}=\hat{W}_{\Theta \Theta} \\
\hat{V} B_{\Theta}^{*}+\hat{U} B^{*}-S^{*} \hat{U}=\frac{1}{\sigma} B_{\Theta \Theta}^{*},
\end{gathered}
$$




$$
2 \hat{U}+\hat{V}_{\Theta}=0,
$$

with the boundary conditions

$$
\hat{W}=1, \hat{U}=\hat{V}=B_{\Theta}^{*}=0 \quad \text { on } \quad \Theta=0,
$$

and

$$
\hat{U} \rightarrow 0, \hat{W} \rightarrow \hat{W}_{e}, B^{*} \rightarrow \hat{W}_{e}^{2} \quad \text { as } \quad \Theta \rightarrow-\infty .
$$

The unsteady form of (1.1)-(1.4), with suitable initial conditions, was derived by DFH for a class of axisymmetric, similarity-type solutions arising in the spin-up of a linearly stratified fluid in a conical container; the reader is referred to this previous work for details of the derivation of (1.1)-(1.4). More general classes of axisymmetric container have been discussed by Hewitt et al. (1997b), in which it was shown that the governing system (away from the rotation axis) is essentially reduced to subsets of the equations above. In this sense the cone is the most interesting simple container to examine.

In the system (1.1)-(1.6), $\hat{U}$ is directly related to the radial velocity component, whilst $\hat{W}$ and $\hat{V}$ are similarly associated with azimuthal and normal boundary-layer velocity components. This notation is consistent with that developed by DFH, and the reader is again referred to that paper for a more complete description of the derivation and non-dimensionalization. The term $B^{*}$ is a non-dimensional measure of the local density perturbation, and $\Theta \in[0,-\infty)$ is a scaled coordinate normal to the boundary. As noted above, we shall make no assumptions concerning the sign of $\hat{W}_{e}$.

The boundary-layer system is governed by three parameters, namely $\left\{\hat{W}_{e}, S^{*}, \sigma\right\}$. Physically, $\hat{W}_{e}$ is the ratio of the fluid rotation rate at the boundary-layer edge to the rotation rate of the container, $S^{*}$ is a slightly redefined Burger number, and $\sigma$ is a Schmidt number. It is worth noting that there is no parameter appearing in (1.1)-(1.4) associated with the apex-angle of the conical container. This dependence has been absorbed into the definitions of $S^{*}$ and $B^{*}$ as described by DFH.

Particular cases of interest are a rotating boundary but stationary fluid $\left(\hat{W}_{e}=0\right.$, analogous to the von Kármán 1921 problem), a small difference in rotation rates of the boundary and far-field fluid ( $\hat{W}_{e} \approx 1$, the linear Ekman boundary layer), a stationary boundary but rotating far-field fluid $\left(1 / \hat{W}_{e} \rightarrow 0\right.$, analogous to the Bödewadt 1940 problem) and counter-rotation of the far-field fluid and boundary $\left(\hat{W}_{e}<0\right)$.

The parameter $S^{*}-\hat{W}_{e}^{2}$ is a measure of the relative importance of buoyancy effects and Coriolis forces, with $\left|S^{*}-\hat{W}_{e}^{2}\right| \gg 1$ corresponding to a strongly stratified fluid. There is a subset of the boundary-layer system that we shall refer to in some detail, namely the case of a homogeneous fluid, $B^{*}=S^{*}=\hat{W}_{e}^{2}$, for which the equations (1.1)-(1.6) reduce to those obtained for rotating disk flows. We can recover the classical rotating disk equations (RDE) with a change of variables,

$$
\left\{\hat{U}, \hat{W}, \hat{V}, B^{*}, \Theta\right\} \rightarrow\left\{f_{z}, g, 2 f, \hat{W}_{e}^{2},-z\right\},
$$

to obtain

$$
\begin{gathered}
f_{z z z}+2 f f_{z z}-f_{z}^{2}+g^{2}-\hat{W}_{e}^{2}=0, \\
g_{z z}+2 f g_{z}-2 g f_{z}=0 .
\end{gathered}
$$

The conditions applied to the system (1.8)-(1.9) are no-slip and impermeability at the disk together with an unchanged rigid-body rotation at infinity,

$$
\begin{aligned}
& f_{z} \rightarrow 0, g \rightarrow \hat{W}_{e} \quad \text { as } \quad z \rightarrow \infty, \\
& f=f_{z}=0, g=1 \quad \text { on } \quad z=0 .
\end{aligned}
$$


We note that the solution for the swirling flow above a rotating disk is an exact solution of the Navier-Stokes equations. This is not the case for the conical container since terms of $O\left(E^{1 / 2} / r\right)$ are neglected when deriving the governing equations and the boundary-layer structure must have some near-apex region.

The governing equations for the steady rotating disk problem appear to be relatively straightforward at first sight, with solutions that depend on a single parameter, namely the ratio of the angular frequency of the fluid at infinity to that of the disk $\left(\hat{W}_{e}\right)$. Nevertheless, as discussed by (amongst many others) Zandbergen (1979) and Dijkstra (1980), the problem has a number of interesting properties. It has been shown that there are regions of parameter space for which no solution can be located, and other regions where non-unique solutions exist. Most attention has been centred near the part of parameter space that corresponds to the fluid being at rest whilst the infinite disk rotates with a non-dimensionalized angular frequency of unity (near $\hat{W}_{e}=0$ in our terminology). In this region an infinite number of solution branches are available. It has been shown by Dijkstra (1980) that the $n$th solution branch consists of an $O(1)$ region immediately adjacent to the disk, followed by $(n-1)$ inviscid cell solutions, which are separated by viscous interlayers. Each time the solution branches, another inviscid cell solution is added to the existing chain; the amplitude of the new cell solution can also be related to that immediately adjacent to it.

Other well known features for the solutions to (1.8)-(1.9) include an infinity of solution branches connected through a singular solution when $\hat{W}_{e}^{2} \approx 2.061$, and a fold in the primary solution branch near $\hat{W}_{e} \approx-0.1605$. This latter value denotes the emergence of a second branch of solution, which can be continued further to provide the higher branches described above. A complete description of the large body of work concerning aspects of the RDE is given in the review article by Zandbergen \& Dijkstra (1987). In what follows we shall use many of the concepts developed for the RDE (which are also applicable to the more general system when $B^{*} \equiv \hat{W}_{e}^{2}=S^{*}$ ) as a starting point for an investigation of the (stratified) boundary-layer system (1.1)-(1.6).

The outline for the paper is as follows. In $\S 2$, we present a number of results concerning steady solutions to the system (1.1)-(1.6). In $\S 2.1$, we show that nonuniform buoyancy solutions arise on $S^{*}=\hat{W}_{e}^{2}$ as imperfect bifurcations from the classical solutions to the RDE. In $\S 3$, we determine boundaries in $\left(S^{*}, \hat{W}_{e}\right)$ parameter space (with $\sigma$ fixed) beyond which steady solution branches cannot be continued. Singular solutions to the RDE have been discussed by a number of authors, Bodonyi (1975), and $\S 3.3$ extends this analysis, deriving a boundary in the $\left(S^{*}, \hat{W}_{e}\right)$ parameter space along which such solutions can be located. In $\S 3.4$ we discuss the limit of small normal velocity at the boundary-layer edge since this limit is often of relevance for steady states near critical boundaries in parameter space. Finally, in $\S 4$ we present some concluding remarks. The Appendix provides details of a non-axisymmetric extension of von Kármán's exact solution for the swirling flow above a rotating disk, which has implications for the existence of nonlinear, non-axisymmetric, steady boundary-layer states for the more general boundary-layer system (1.1)-(1.6).

\section{Steady boundary-layer states on $S^{*}=\hat{W}_{e}^{2}$.}

When $S^{*}=\hat{W}_{e}^{2}$ with $B^{*} \equiv \hat{W}_{e}^{2}$, steady states of the RDE (1.8)-(1.11) are also solutions of the more general system (1.1)-(1.6). Thus, on this surface in parameter space, there exist solutions that have a velocity field equivalent to that obtained for the rotating disk problem, with a uniform $B^{*}$-profile; the number of such solutions 


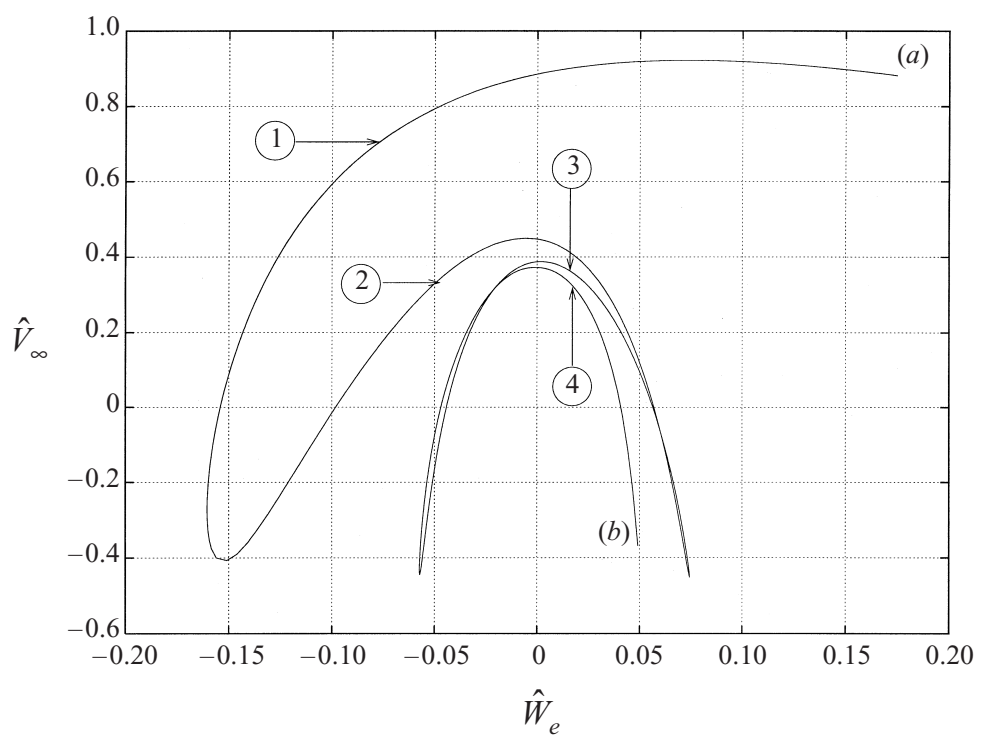

FIGURE 2. The first four solution branches to the rotating disk equations (near $\hat{W}_{e}=0$ ).

depends on the precise value of $\hat{W}_{e}$, as illustrated by figure 2 . The results shown in this figure agree with those presented by other authors, see for example Zandbergen (1979).

The solution branches shown in figure 2 were obtained via a finite difference approach in which either $\hat{W}_{e}$, or

$$
\int_{0}^{-\infty}\left(\hat{V}(\bar{\Theta})-\hat{V}_{\infty}\right) d \bar{\Theta}
$$

is specified. Here, $\hat{V}_{\infty}$ is the value, at the boundary-layer edge, of the velocity component perpendicular to the disk/container wall. Switching between these two parameters where appropriate allows the solution to be continued beyond the limit points shown in figure 2. Later computations were performed using an orthogonal collocation method together with arc-length continuation. This approach was applied by the package AUTO (Doedel \& Wang 1995), which allows for numerical continuation of solutions when varying any of the parameters of the problem.

Solving (1.1)-(1.6), rather than (1.8)-(1.11), can cause problems since (as we shall see) the uniform buoyancy solution can be numerically unstable when $\hat{V}_{\infty}<0$; however, forcing the buoyancy to remain uniform in the computational method removes this difficulty. A more detailed discussion of the large- $\Theta$ behaviour of the system (1.1)-(1.6) will be presented later; however, for $S^{*}=\hat{W}_{e}^{2}$ we note that solutions with non-uniform $B^{*}$-profiles must satisfy

$$
B^{*} \sim b_{0} \exp \left(\sigma \hat{V}_{\infty} \Theta\right) \text { for }|\Theta| \gg 1
$$

where $b_{0}$ is a constant. Therefore $\hat{V}_{\infty}<0$ leads to an unacceptable, exponentially growing solution far from the boundary since $\Theta \in[0,-\infty)$.

Higher branch solutions to the RDE (beyond those shown in figure 2) can be obtained with the same procedure by continuing the computations beyond the point (b) shown in the figure. Each time the solution branches another inviscid cell is introduced into the boundary-layer solution, as described by Dijkstra (1980), and 


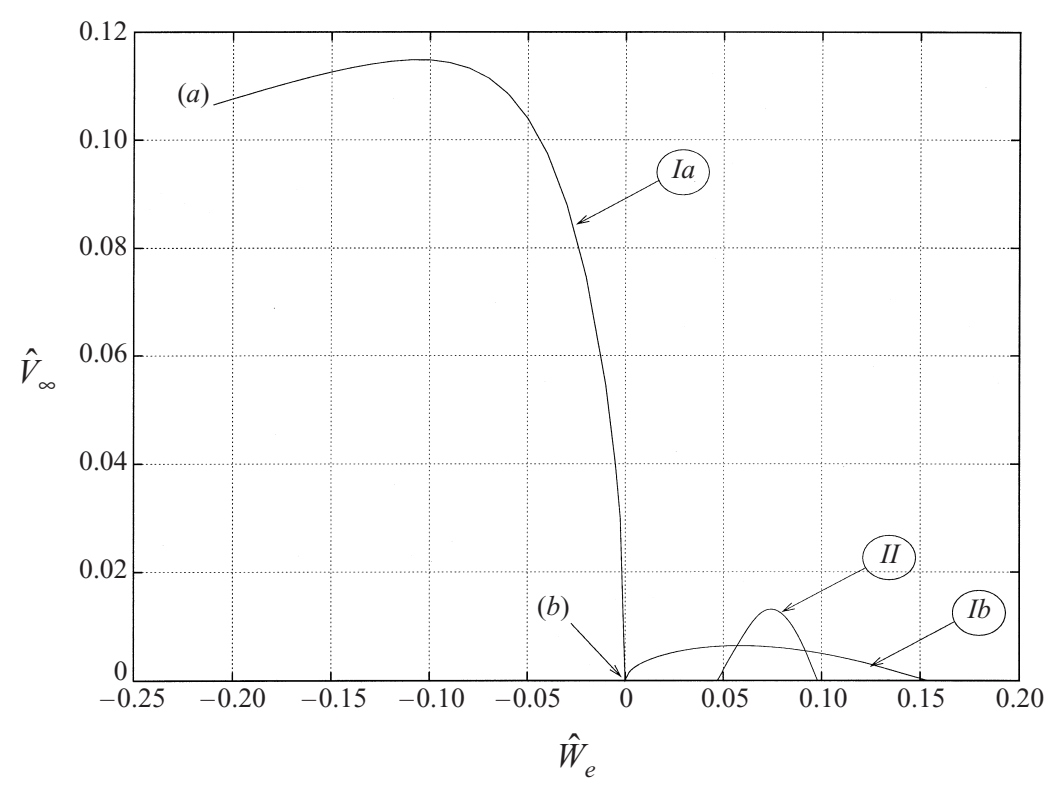

FIGURE 3. Some of the non-uniform buoyancy solutions that are available on $S^{*}=\hat{W}_{e}^{2}$, with $\sigma=1$.

thus a larger domain is required in the computation with a corresponding increase in the necessary number of grid points.

\subsection{A bifurcation to states of non-uniform buoyancy on $S^{*}=\hat{W}_{e}^{2}$}

At general values of the parameter $\hat{W}_{e}$ (maintaining the restriction $S^{*}=\hat{W}_{e}^{2}$ ) there exist other states for which $B^{*}$ is non-uniform throughout the boundary layer; some of these solution branches are shown in figure 3 for $\sigma=1$. In figure 3 , branch (Ia) bifurcates from the branch-1 rotating disk solution, whilst branch (II) appears as a bifurcation from the branch-2 rotating disk solution (see figures 4 and 5). Branch $(I a)$ can be continued beyond point $(a)$ up to a critical value of $\hat{W}_{e}$ (at which point $\left.\hat{V}_{\infty}=0\right)$ and $(b)$ is a $\left(\left|\hat{W}_{e}\right| \ll 1\right)$ region that we shall discuss in detail later. The solutions displayed in figure 3 correspond to states that have a density variation throughout the boundary-layer, matching to an interior swirling flow that is of uniform density.

Locating solutions such as those shown in figure 3 can be difficult in general. Some of the non-uniform branches can be found for $S^{*}=\hat{W}_{e}^{2}$ by (numerically) obtaining a higher branch steady solution at a general point in the parameter space and then following this solution in the limit $S^{*} \rightarrow \hat{W}_{e}^{2}$. A more systematic method is available however since there are critical values of $\hat{W}_{e}$ on the solution branches of the rotating disk equations, which when used as a starting point for a perturbation analysis of (1.1)-(1.4), allow for both rotating-disk solutions and states with a weak density variation. The values of $\hat{W}_{e}$ where these bifurcation points are located can be derived from the following expansion procedure:

$$
\begin{gathered}
\hat{W}_{e}=W_{t c}+\delta, \quad|\delta| \ll 1, \\
S^{*}=W_{t c}^{2}+2 \delta W_{t c}+\delta^{2},
\end{gathered}
$$


Steady boundary-layer solutions for a swirling stratified fluid in a cone

$$
B^{*}=W_{t c}^{2}+\delta B_{1}(\Theta)+\cdots,
$$

$$
(\hat{U}, \hat{V}, \hat{W})^{T}=\left(U_{0}(\Theta), V_{0}(\Theta), W_{0}(\Theta)\right)^{T}+\delta\left(U_{1}(\Theta), V_{1}(\Theta), W_{1}(\Theta)\right)^{T}+\cdots,
$$

where $\hat{W}_{e}=W_{t c}$ is the critical parameter value at which the bifurcation occurs. Thus, we are perturbing about a point on $S^{*}=\hat{W}_{e}^{2}$ and looking for solutions that have weakly non-uniform $B^{*}$-profiles.

At leading order, $O\left(\delta^{0}\right)$, after substitution of the above expansions into the governing system, we obtain the RDE, but in the form

$$
\begin{gathered}
U_{0}^{2}+V_{0} U_{0}^{\prime}-W_{0}^{2}=U_{0}^{\prime \prime}-W_{t c}^{2}, \\
2 U_{0} W_{0}+V_{0} W_{0}^{\prime}=W_{0}^{\prime \prime}, \\
2 U_{0}+V_{0}^{\prime}=0,
\end{gathered}
$$

with $U_{0}=V_{0}=0, W_{0}=1$ on $\Theta=0$ and $U_{0} \rightarrow 0, W_{0} \rightarrow W_{t c}$ as $\Theta \rightarrow-\infty$. Obviously, $W_{t c}$ is not determined at this stage of the expansion. Solutions of this system for an unrestricted far-field rotation rate $\left(\hat{W}_{e}\right)$ are given in figure 2 in the neighbourhood of $\hat{W}_{e}=0$.

At next order, $O(\delta)$, we obtain

$$
\begin{gathered}
2 U_{0} U_{1}+V_{0} U_{1}^{\prime}+V_{1} U_{0}^{\prime}-2 W_{0} W_{1}=U_{1}^{\prime \prime}-B_{1}, \\
2\left(U_{0} W_{1}+U_{1} W_{0}\right)+V_{0} W_{1}^{\prime}+V_{1} W_{0}^{\prime}=W_{1}^{\prime \prime}, \\
V_{0} B_{1}^{\prime}+U_{0} B_{1}-2 W_{t c} U_{0}=\sigma^{-1} B_{1}^{\prime \prime}, \\
2 U_{1}+V_{1}^{\prime}=0,
\end{gathered}
$$

with $U_{1}=V_{1}=W_{1}=B_{1}^{\prime}=0$ on $\Theta=0$ and $U_{1} \rightarrow 0, W_{1} \rightarrow 1, B_{1} \rightarrow 2 W_{t c}$ as $\Theta \rightarrow-\infty$. The equation governing $B_{1}$ can be solved separately in conjunction with the $O\left(\delta^{0}\right)$ (rotating disk) system. Making the substitution,

$$
B_{1}=\bar{B}_{1}+2 W_{t c}
$$

reduces (2.12) to

$$
V_{0} \bar{B}_{1}^{\prime}+U_{0} \bar{B}_{1}=\sigma^{-1} \bar{B}_{1}^{\prime \prime},
$$

with $\bar{B}_{1}^{\prime}=0$ on $\Theta=0$ and $\bar{B}_{1} \rightarrow 0$ as $\Theta \rightarrow-\infty$. Thus (2.15) together with (2.7)-(2.9) forms an eigenvalue problem, and thus determines the bifurcation point $W_{t c}$.

It is a straightforward process to solve (2.15) by a shooting method (from $\Theta=0$ to $\Theta \rightarrow-\infty)$ given any leading-order solution $\left\{U_{0}, V_{0}, W_{0}\right\}$. Since $(2.15)$ is linear we may arbitrarily specify $\bar{B}_{1}(\Theta=0)=1$, then by coupling the shooting routine to a solution of the $O\left(\delta^{0}\right)$ system we may iterate on $W_{t c}$ to satisfy the remaining boundary condition $\bar{B}_{1} \rightarrow 0$ as $\Theta \rightarrow-\infty$. Application of this procedure shows that for a non-trivial solution to $(2.15)$ to exist, the rotation rate (for a branch-1, $O\left(\delta^{0}\right)$, leading-order solution) is $W_{t c} \approx-0.1487$ when $\sigma=1$. In terms of the figures, this value of $W_{t c}$ corresponds to the cross-over of the solution branch (Ia) from figure 2 and branch-1 from figure 1, as shown in figure 3.

The dependence of the bifurcation point on $\sigma$ is obviously of some importance. In particular one may ask if there is always a bifurcation to a non-uniform buoyancy solution (e.g. for the branch-1 solutions to the RDE), or is there some finite range of $\sigma$ within which this feature can be located. The existence of the bifurcation point is obviously crucial for the existence of steady states in the $\hat{W}_{e}<0$ region (see figure 4). In table 1 we show how the location of the bifurcation point $\left(W_{t c}\right)$ varies with the 


$\begin{array}{cccc}\sigma & W_{t c} & \sigma & W_{t c} \\ 1 & -0.1487 & 16 & -0.1523 \\ 2 & -0.1491 & 32 & -0.1527 \\ 4 & -0.1504 & 64 & -0.1530 \\ 8 & -0.1516 & 128 & -0.1531\end{array}$

TABLE 1. Variation of the first bifurcation point with $\sigma$.

Schmidt number $\sigma$; as noted earlier, $W_{t c} \approx-0.1487$ when $\sigma=1$. As can be seen from the table of values, the bifurcation persists at large values of $\sigma$, and $W_{t c}$ asymptotes to a finite value.

The same routine may be applied to the higher branch solutions of the rotating disk problem to obtain other values for $W_{t c}$; in this way we can locate other non-uniform solutions, such as that denoted by $(I I)$ in figure 3 .

The solution of (2.15) determines the location of the bifurcation point; however, since this equation for $\bar{B}_{1}$ is linear we must continue the expansion procedure in order to determine an 'amplitude' for the bifurcated solution. It is easy to see that a general solution to the $O(\delta)$ system can be written as

$$
\begin{aligned}
U_{1} & =U_{1 H}+A \tilde{U}_{1}, \\
V_{1} & =V_{1 H}+A \tilde{V}_{1}, \\
W_{1} & =W_{1 H}+A \tilde{W}_{1}, \\
B_{1} & =2 W_{t c}+A \tilde{B}_{1},
\end{aligned}
$$

where $A$ is a real constant (representing some amplitude measure) that remains undetermined at this order. We note that $\left\{\tilde{U}_{1}, \tilde{V}_{1}, \tilde{W}_{1}\right\}$ satisfy (2.10)-(2.11), (2.13) but with $\left\{U_{1}, V_{1}, W_{1}\right\} \rightarrow\left\{\tilde{U}_{1}, \tilde{V}_{1}, \tilde{W}_{1}\right\}$ and the changed boundary condition $\tilde{W}_{1} \rightarrow 0$ as $\Theta \rightarrow-\infty$.

The solution parts $\left\{U_{1 H}, V_{1 H}, W_{1 H}\right\}$ represent the first correction term in a Taylor series expansion of the rotating disk solution about the critical point, that is

$$
U_{1 H}=\left.\frac{\partial U_{0}}{\partial \hat{W}_{e}}\right|_{\hat{W}_{e}=W_{t c}}, \quad V_{1 H}=\left.\frac{\partial V_{0}}{\partial \hat{W}_{e}}\right|_{\hat{W}_{e}=W_{t c}}, \quad W_{1 H}=\left.\frac{\partial W_{0}}{\partial \hat{W}_{e}}\right|_{\hat{W}_{e}=W_{t c}} .
$$

To determine the constant $A$ we must continue the above expansion to $O\left(\delta^{2}\right)$, at which point the density transport equation can again be solved in isolation given the solutions (2.16)-(2.19). Substitution of the expansions (2.3)-(2.6) leads to

$$
V_{0} B_{2}^{\prime}+V_{1} B_{1}^{\prime}+U_{0} B_{2}+U_{1} B_{1}-2 W_{t c} U_{1}-U_{0}=\sigma^{-1} B_{2}^{\prime \prime},
$$

with boundary conditions $B_{2}^{\prime}=0$ on $\Theta=0$ and $B_{2} \rightarrow 1$ as $\Theta \rightarrow-\infty$. Substitution of the solutions (2.16)-(2.19) into (2.21), together with (2.14) and the redefinition

$$
B_{2}=\bar{B}_{2}+1 \text {, }
$$

leads to

$$
V_{0} \bar{B}_{2}^{\prime}+U_{0} \bar{B}_{2}-\frac{1}{\sigma} \bar{B}_{2}^{\prime \prime}=-A\left(V_{1 H} \tilde{B}_{1}^{\prime}+U_{1 H} \tilde{B}_{1}\right)-A^{2}\left(\tilde{V}_{1} \tilde{B}_{1}^{\prime}+\tilde{U}_{1} \tilde{B}_{1}\right)
$$

with $\bar{B}_{2}^{\prime}=0$ on $\Theta=0$ and $\bar{B}_{2} \rightarrow 0$ as $\Theta \rightarrow-\infty$. 
Since the homogeneous form of (2.23) has a non-trivial solution, an orthogonality condition must be satisfied for the inhomogeneous equation to have a solution. Hence we determine the amplitude constant, $A$, from the solvability condition

$$
A^{2} a_{2}+A a_{1}=0 ;
$$

the real constants $a_{1}$ and $a_{2}$ are easily shown to be

$$
\begin{gathered}
a_{1}=\int_{\Theta=0}^{-\infty} \Phi(\Theta)\left\{V_{1 H}(\Theta) \tilde{B}_{1}^{\prime}(\Theta)+U_{1 H}(\Theta) \tilde{B}_{1}(\Theta)\right\} \mathrm{d} \Theta, \\
a_{2}=\int_{\Theta=0}^{-\infty} \Phi(\Theta)\left\{\tilde{V}_{1}(\Theta) \tilde{B}_{1}^{\prime}(\Theta)+\tilde{U}_{1}(\Theta) \tilde{B}_{1}(\Theta)\right\} \mathrm{d} \Theta,
\end{gathered}
$$

where $\Phi(\Theta)$ corresponds to the adjoint of the homogeneous form of (2.23). Thus there are two solution branches that correspond to $A=0$ (the rotating disk solution) and $A=-a_{1} / a_{2}$ (the weakly non-uniform buoyancy solution).

Rather than solving the adjoint problem and computing the integrals to give $a_{1,2}$ we take a slightly more direct approach. We solve the leading-order rotating disk system (2.7)-(2.9) in conjunction with the eigenvalue problem (2.15) to obtain $\tilde{B}_{1}$ and $W_{t c}$, then the terms $\left\{U_{1 H}, V_{1 H}, W_{1 H}\right\}$ are obtained directly and $\left\{\tilde{U}_{1}, \tilde{V}_{1}, \tilde{W}_{1}\right\}$ can be determined by solving the discretized system resulting from a central differencing scheme. The amplitude constant $A$ is then determined by an iteration scheme applied directly to (2.23), solved by the same shooting approach applied to (2.15). For the specific example given above we find

$$
\text { for } \sigma=1: \quad W_{t c} \approx-0.1487, \quad A \approx 0.18045 .
$$

We should note at this stage that not all states available for $S^{*}=\hat{W}_{e}^{2}$ arise as bifurcations from the rotating disk solutions. In particular, as $\sigma$ is increased it is possible to locate other states (again with non-uniform $B^{*}$-profiles) that are isolated from the rotating disk solutions.

\subsubsection{An imperfect bifurcation}

The behaviour of the state loci in the $\left\{\hat{V}_{\infty}, \hat{W}_{e}\right\}$-plane (for $S^{*}=\hat{W}_{e}^{2}, \hat{W}_{e}^{2} \pm 10^{-3}$, with $\sigma=1$ ) is shown in figures $4 \& 5$ for specific cases. It is clear that the bifurcation described above is structurally unstable when the modified Burger number, $S^{*}$, is perturbed away from $S^{*}=\hat{W}_{e}^{2}$. Therefore, we may anticipate that $S^{*}-\hat{W}_{e}^{2}$ acts as an imperfection when considering steady states near to the bifurcation point $\hat{W}_{e}=W_{t c}$, $S^{*}=W_{t c}^{2}$

To discuss this more general case, we can perturb about $\hat{W}_{e}=W_{t c}$ as before, but to introduce the imperfection we must also perturb $S^{*}$ such that $S^{*}-W_{t c}^{2}=O\left(\delta^{2}\right)$; in this approach, additional terms will contribute to the final solvability condition. On writing

$$
\begin{gathered}
\hat{W}_{e}=W_{t c}+\delta, \quad|\delta| \ll 1, \\
S^{*}=W_{t c}^{2}+2 \delta W_{t c}+(1+\tilde{S}) \delta^{2},
\end{gathered}
$$

where $\tilde{S}=O(1)$, the development of the expansion proceeds as before until, at $O\left(\delta^{2}\right)$, (2.21) becomes

$$
V_{0} B_{2}^{\prime}+V_{1} B_{1}^{\prime}+U_{0} B_{2}+U_{1} B_{1}-2 W_{t c} U_{1}-(1+\tilde{S}) U_{0}=\sigma^{-1} B_{2}^{\prime \prime},
$$




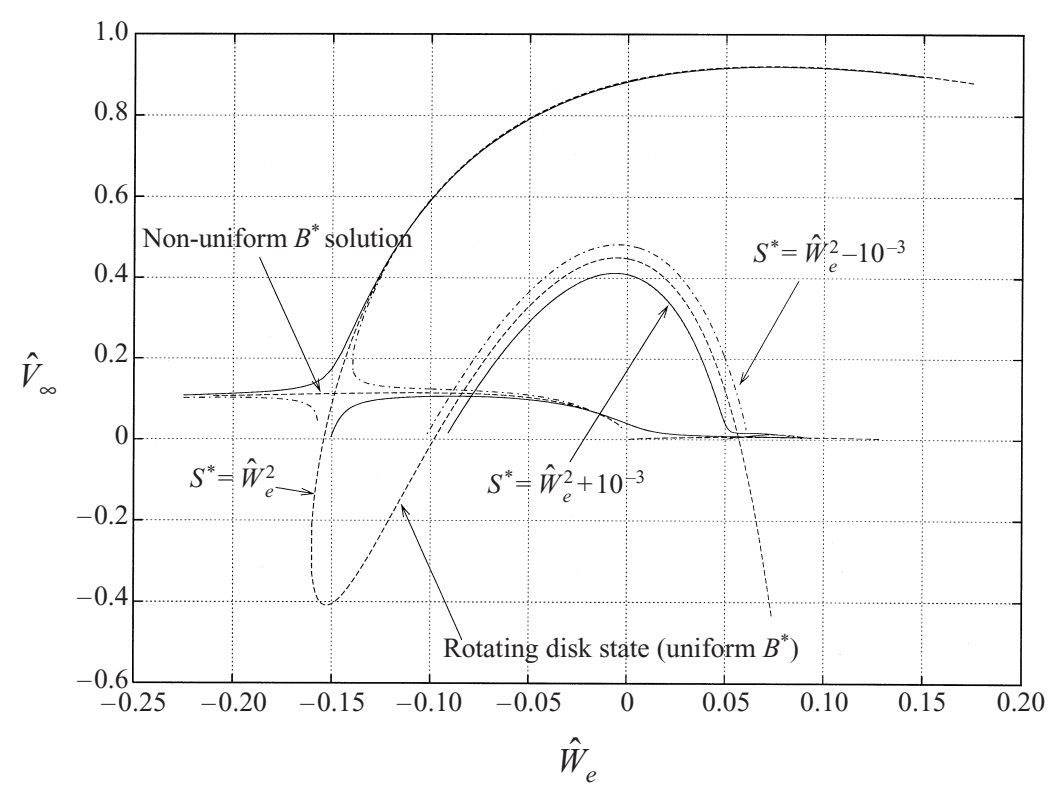

FigURE 4. Some steady solutions for $\sigma=1$, and $S^{*}=\hat{W}_{e}^{2}, \hat{W}_{e}^{2} \pm 10^{-3}$. Notice the bifurcation point near $\hat{W}_{e} \approx-0.1487$.

or equivalently

$$
V_{0} \bar{B}_{2}^{\prime}+U_{0} \bar{B}_{2}-\frac{1}{\sigma} \bar{B}_{2}^{\prime \prime}=-A\left(V_{1 H} \tilde{B}_{1}^{\prime}+U_{1 H} \tilde{B}_{1}\right)-A^{2}\left(\tilde{V}_{1} \tilde{B}_{1}^{\prime}+\tilde{U}_{1} \tilde{B}_{1}\right)+\tilde{S} U_{0}
$$

Again $A$ must satisfy a solvability condition, which in this case is of the form

$$
A^{2} a_{2}+A a_{1}+\tilde{S} a_{0}=0,
$$

where $a_{1}$ and $a_{2}$ are as defined in the previous approach, and

$$
a_{0}=\int_{\Theta=0}^{\infty} \Phi(\Theta) U_{0}(\Theta) \mathrm{d} \Theta .
$$

Therefore

$$
A=\frac{-a_{1} \pm\left(a_{1}^{2}-4 \tilde{S} a_{2} a_{0}\right)^{1 / 2}}{2 a_{2}},
$$

leading to the previous result (2.24) when $\tilde{S}=0$. However, there is a critical perturbation such that no solutions (see figure 7) to (1.1)-(1.4) are available in this neighbourhood of the rotating disk solution when $S^{*}=W_{t c}^{2}+\delta^{2} \tilde{S}$, with

$$
\tilde{S}>\tilde{S}_{\text {crit }} \equiv \frac{a_{1}^{2}}{4 a_{2} a_{0}} .
$$

In figure 6(a) we show the behaviour of the amplitude constant $A$ with the imperfection parameter $\tilde{S}$. Rather than computing the adjoint function and the constants $a_{0}, a_{1}$ and $a_{2}$ we again iterate directly on the solution of (2.30). As noted above, when $\tilde{S}=0$ the two solutions are $A=0$ and $A \approx 0.18$ (the solution with non-uniform $B^{*}$-profiles). The critical value of $\tilde{S}=\tilde{S}_{c r i t} \approx-13.21$, as defined by (2.34), is clearly shown. We can interpret this critical value as a fold of the surface in parameter space that defines the steady states. For example, as shown in figure 


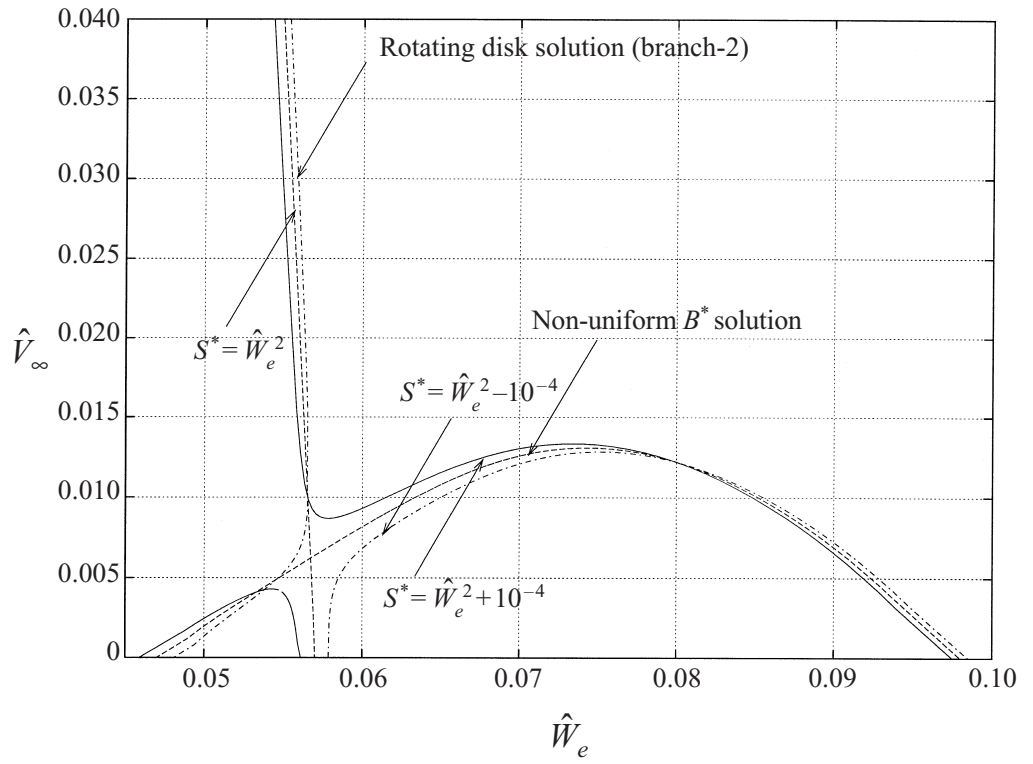

FIGURE 5. The solution branches in the region $\hat{W}_{e} \in[0.05,0.1]$ with $S^{*}=\hat{W}_{e}^{2}, \hat{W}_{e}^{2} \pm 10^{-4}$ and $\sigma=1$. A further solution arises as a bifurcation from the branch-2 rotating disk solution.
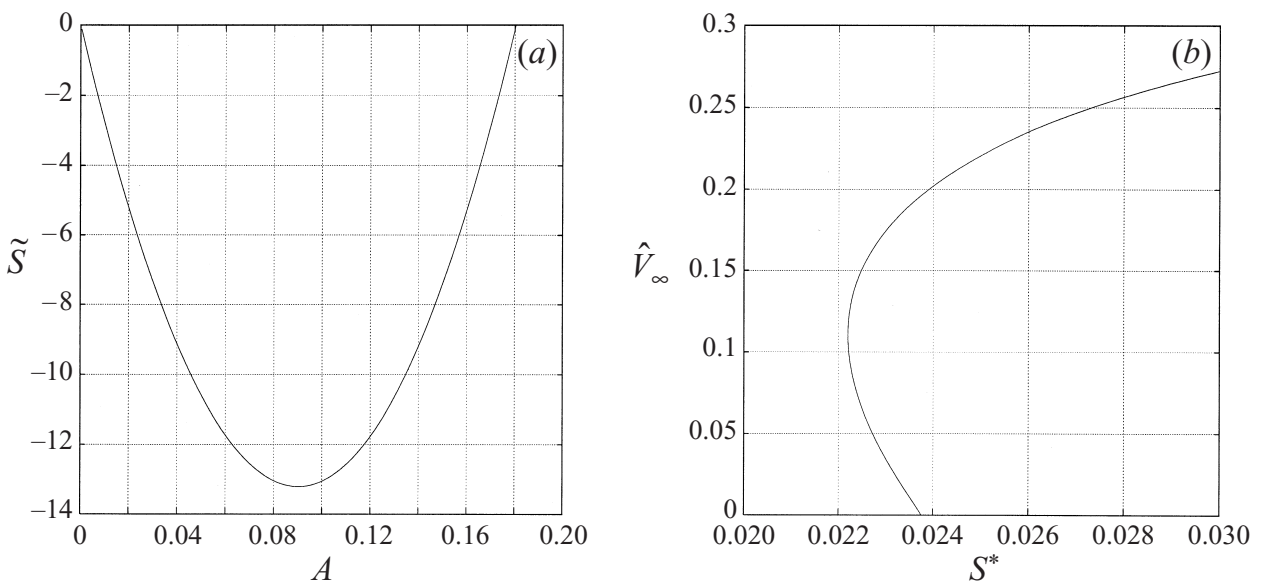

FiguRE 6. (a) The amplitude of the bifurcated solution (near $\hat{W}_{e}=W_{t c}$ ) as a function of the imperfection parameter $\tilde{S}$. Note that there is a critical value $\tilde{S}_{c r i t} \approx-13.21$. (b) The critical value $\tilde{S}_{c r i t}$ is associated with a fold in the solution surface, as shown here for $\hat{W}_{e}=-0.149$, the fold is at $S^{*}=0.0222$

$6(b)$ with $\hat{W}_{e}=-0.149$, continuation of the branch-1 steady state shows a fold at $S^{*}=S_{\text {fold }}^{*}=0.0222$ (to four decimal places), which is also the value given by the local description (as provided by the above expansion)

$$
S_{\text {fold }}^{*}=\hat{W}_{e}^{2}+\tilde{S}_{\text {crit }}\left(\hat{W}_{e}-W_{t c}\right)^{2},
$$

to this accuracy.

A similar bifurcation analysis can be applied to the RDE to search for nonaxisymmetric solutions of von Kármán similarity form. In the Appendix we present details of a bifurcated solution branch corresponding to an exact solution of the 


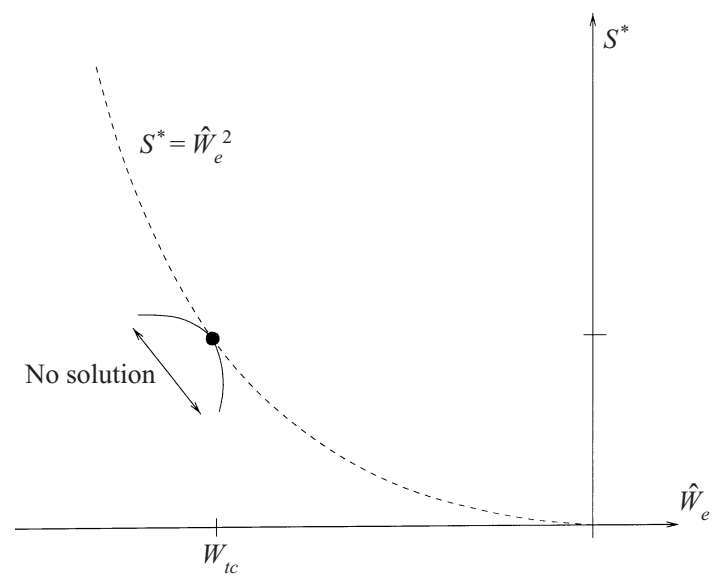

FIGURE 7. A schematic view of the $\left(S^{*}, \hat{W}_{e}\right)$-plane near $\hat{W}_{e}=W_{t c}$. The solid lines represent the set of one-sided bifurcation points near to the critical value $W_{e}=W_{b i f}$. The local analysis determines this set to be $S^{*}=W_{t c}^{2}+a_{1}^{2}\left(\hat{W}_{e}-W_{t c}\right)^{2} / 4 a_{2} a_{0}$.

Navier-Stokes equations for a non-axisymmetric flow above a rotating disk; the axisymmetric boundary conditions are maintained in this flow. This result will hopefully provide a starting point for a continued discussion of the conical/stratified boundarylayer problem extended to include non-axisymmetric flows. (Experimental, unsteady, non-axisymmetric flow evolutions have been discussed by HDDF for the spin-down of stratified fluid in a conical container.)

\section{Critical boundaries in parameter space}

Much of our motivation for the present analysis arises from an attempt to understand the evolution of time-dependent 'spin-up/down' processes for a linearly stratified fluid in a container with sloping walls. Obviously, an ability to classify the whole parameter space in terms of the steady states that exist is invaluable both for choosing parameter values for experimentation and when considering the stability of these states. As has been seen in the previous papers of DFH, and HDDF, boundaries in the $\left(S^{*}, \hat{W}_{e}\right)$-plane (with $\sigma$ fixed) beyond which steady states cannot be continued can play a crucial role in parameter-space diagrams used to interpret the readjustment process of spin-up/down.

The behaviour of the solution branches near existence boundaries can be classified into three main groups, namely folds, singular solutions and states for which $\hat{V}_{\infty} \rightarrow 0$ on approaching the critical boundary. As noted by HDDF, for fixed $\sigma$ the solution branches are best considered as a surface in $\left\{S^{*}, \hat{W}_{e}, \hat{V}_{\infty}\right\}$-space. Folds in this surface occur, in general, at finite values of $\hat{V}_{\infty}$ and can only be obtained (globally) by numerical solution of the governing boundary value problem. We can however give some details concerning the other two cases, namely singular solutions and cases for which $\left|\hat{V}_{\infty}\right| \ll 1$. Before presenting descriptions of these two regimes we shall give a brief discussion of the large- $|\Theta|$ form of available solutions. We shall also discuss the region $(b),\left|\hat{W}_{e}\right| \ll 1$, as shown in figure 3 .

For the rotating disk system, that is (1.1)-(1.6) with $B^{*} \equiv \hat{W}_{e}^{2}=S^{*}$, the appropriate large- $|\Theta|$ form can be determined by writing $\hat{U}=u(\Theta), \hat{V}=\hat{V}_{\infty}+v(\Theta), \hat{W}=\hat{W}_{e}+$ $w(\Theta)$ and then neglecting nonlinear terms of $u, v$ and $w$. The relevant exponential 
form of the unknowns can then be determined after some algebra, and has been given by Rogers \& Lance (1960). In our more general case, following the above procedure together with writing $B^{*}=\hat{W}_{e}^{2}+b(\Theta)$, we find that assuming the exponential form

$$
\{u(\Theta), v(\Theta), w(\Theta), b(\Theta)\}^{T}=\left\{u_{0}, v_{0}, w_{0}, b_{0}\right\}^{T} \exp (\lambda \Theta), \quad|\Theta| \gg 1,
$$

where $u_{0}, v_{0}, w_{0}$ and $b_{0}$ are constants, leads to the polynomial

$$
\begin{aligned}
& \lambda^{5}-\hat{V}_{\infty}(2+\sigma) \lambda^{4}+\hat{V}_{\infty}^{2}(1+2 \sigma) \lambda^{3}-\sigma \hat{V}_{\infty}^{3} \lambda^{2} \\
& \quad+\left(4 \hat{W}_{e}^{2}-\sigma\left[\hat{W}_{e}^{2}-S^{*}\right]\right) \lambda-\sigma \hat{V}_{\infty}\left(3 \hat{W}_{e}^{2}+S^{*}\right)=0 .
\end{aligned}
$$

If $\lambda_{1}$ is the smallest positive root of this equation, then the multiplying constants must satisfy

$$
\begin{gathered}
\frac{w_{0}}{b_{0}}=2 \hat{W}_{e} /\left(\lambda_{1}^{4}-2 \hat{V}_{\infty} \lambda_{1}^{3}+\hat{V}_{\infty}^{2} \lambda_{1}^{2}+4 \hat{W}_{e}^{2}\right), \\
\frac{u_{0}}{w_{0}}=\left(\lambda_{1}^{2}-\hat{V}_{\infty} \lambda_{1}\right) / 2 \hat{W}_{e},
\end{gathered}
$$

and (from the continuity of mass condition) $v_{0} / u_{0}=-2 / \lambda_{1}$. This description has been checked against numerical results for a number of the solution branches obtained at general parameter values. We note that, in all the cases for which we have obtained a numerical solution with $B^{*} \neq \hat{W}_{e}^{2}$, the edge behaviour has been such that $\hat{V}_{\infty}>0$. At general values of $S^{*} \neq \hat{W}_{e}^{2}$ we cannot rule out that a solution of the full equations is possible with $\lambda_{1}>0$ and $\hat{V}_{\infty}<0$; however we have been unable to locate any such states.

\subsection{The region $\left|\hat{W}_{e}\right| \ll 1$}

The large- $|\Theta|$ behaviour described by (3.2) is only strictly valid for solutions with $B^{*} \neq \hat{W}_{e}^{2}, \hat{W}_{e} \neq 0$. When $\hat{W}_{e}=0$, with a general $S^{*} \neq 0$ we find that the appropriate large- $|\Theta|$ scalings are

$$
\begin{aligned}
& \hat{V} \sim \hat{V}_{\infty}, \text { and }\left\{\hat{U}, \hat{W}, B^{*}\right\}^{T} \sim\left\{u_{0} \exp \left(2 \hat{V}_{\infty} \Theta\right),\right. \\
&\left.w_{0} \exp \left(\hat{V}_{\infty} \Theta\right), \quad b_{0} \exp \left(2 \hat{V}_{\infty} \Theta\right)\right\}^{T},
\end{aligned}
$$

where $\left\{u_{0}, w_{0}, b_{0}\right\}^{T}$ are constants, which satisfy the relations

$$
\begin{gathered}
\frac{u_{0}}{b_{0}}=2 \hat{V}_{\infty}^{2}\left(1-\frac{2}{\sigma}\right) / S^{*}, \\
\frac{w_{0}^{2}}{b_{0}}=1-2 \hat{V}_{\infty}^{2} \frac{u_{0}}{b_{0}} .
\end{gathered}
$$

As we have already noted (with reference to figure 3 ), the origin of the $\left(S^{*}, \hat{W}_{e}\right)$ plane is also a special case. For some solution branches, on approaching the origin $\left(S^{*}=\hat{W}_{e}=0\right)$ it is possible to obtain states that have $\hat{V}_{\infty} \rightarrow 0$ but which are not described by the general $\left(\hat{V}_{\infty} \ll 1\right)$ asymptotic analysis that we shall present in the next section. In this special case we can find three separate large- $|\Theta|$ forms for the boundary-layer system (1.1)-(1.6).

$|\Theta| \gg 1$ behaviour when $\left|\hat{W}_{e}\right| \ll 1$ : Case (i)

Here, we balance the $\hat{W}$ and $B^{*}$ terms in the $\hat{U}$-momentum equation by assuming that the solution has the following behaviour for large, negative $\Theta$ :

$$
\hat{U} \rightarrow U_{0} / \Theta^{2}, \quad \hat{V} \rightarrow 2 U_{0} / \Theta, \quad B^{*} \rightarrow B_{0} / \Theta^{2 \gamma}, \quad \hat{W} \rightarrow W_{0} / \Theta^{\gamma},
$$


where $\gamma<2$ is necessary for self-consistency. The equations for the coefficients $U_{0}$, $W_{0}, B_{0}$ (all assumed to be non-zero for consistency) are found to be

$$
\begin{gathered}
W_{0}^{2}=B_{0}, \\
2 U_{0}(1-\gamma)=\gamma(\gamma+1), \\
2 U_{0}(-2 \gamma)+U_{0}=\frac{2 \gamma}{\sigma}(2 \gamma+1) .
\end{gathered}
$$

Combining the above leads to the following condition for $\gamma$ :

$$
\gamma^{2}(8-4 \sigma)+\gamma(-3 \sigma-4)+\sigma-4=0 .
$$

To maintain a self-consistent description we need roots to (3.12) that satisfy the requirement that $\gamma<2$. It is easy to see that when $\sigma \in[0,20 / 21] \cup[4, \infty)$ there exists a single root of the polynomial (3.12) with the property $0<\gamma<2$.

Allowing for a perturbation about the origin of parameter space, for example by considering the solution on the curve $S^{*}=\hat{W}_{e}^{2}$ as $\hat{W}_{e} \rightarrow 0$, we have $\hat{W}_{e}=\epsilon \ll 1$ and $S^{*}=\epsilon^{2}$. Obviously, the form of decay for $B^{*}$ and $\hat{W}$ (at large values of $\Theta$ ) suggests an outer layer is required when $B^{*} \sim \epsilon^{2}$. This outer layer is defined through the scaled coordinate $\tilde{\Theta}=\Theta \epsilon^{1 / \gamma}$, and leads to

$$
\left.\begin{array}{l}
\hat{U}=\epsilon^{2 / \gamma} \tilde{U}(\tilde{\Theta})+\cdots, \quad \hat{V}=\epsilon^{1 / \gamma} \tilde{V}(\tilde{\Theta})+\cdots, \\
\hat{W}=\epsilon \tilde{W}(\tilde{\Theta})+\cdots, \quad B^{*}=\epsilon^{2} \tilde{B}(\tilde{\Theta})+\cdots
\end{array}\right\}
$$

We shall restrict attention to the case $S^{*}=\hat{W}_{e}=0$ since outer regions can be discussed for each of the following cases in a similar manner.

$|\Theta| \gg 1$ behaviour when $\left|\hat{W}_{e}\right| \ll 1$ : Case (ii)

In this case we balance the $\hat{U}$ and $B^{*}$ terms in the $\hat{U}$-momentum equation by assuming the large- $|\Theta|$ form

$$
\begin{gathered}
\hat{U} \rightarrow U_{0} / \Theta^{2}, \quad \hat{V} \rightarrow 2 U_{0} / \Theta, \\
B^{*} \rightarrow B_{0} / \Theta^{4}, \quad \hat{W} \rightarrow W_{0} / \Theta^{\beta} .
\end{gathered}
$$

This type of decaying behaviour leads to the following equation to determine $\beta$ :

$$
\beta^{2}+\beta\left(1+2 U_{0}\right)-2 U_{0}=0,
$$

where $U_{0}=-20 / 7 \sigma, B_{0}=3 U_{0}^{2}+6 U_{0}$ and $W_{0}$ remains undetermined at this order. For the appropriate balance of the $\hat{U}$-momentum equation we require that $\beta \geqslant 2$. There are two relevant roots to (3.16) for $U_{0} \leqslant-3 / 2-\sqrt{2} \equiv U_{0}^{*}$. At $U_{0}=U_{0}^{*}$, the two $\beta$ values come together at $1+\sqrt{2}$, then as $U_{0}$ decreases below $U_{0}^{*}$, the two values of $\beta$ separate, attaining values of 2 and 3 at $U_{0}=-3$. Since the appropriate root would correspond to the slowest decaying solution at large $|\Theta|$, the smaller value of $\beta$ is the correct choice. This smaller root attains the value of 2 , where $U_{0}=-3$ and $\sigma=20 / 21$, which means that this solution connects smoothly to the structure given as Case (i) above. Therefore, the solution domain is constituted by

$$
\frac{20}{21}<\sigma<\frac{40}{7}(3-2 \sqrt{2}),
$$

and the appropriate choice of $\beta$ is the smaller root of (3.16).

We note that the other obvious scaling at large $|\Theta|$, which involves a balance between $\hat{U}$ and $\hat{W}$ terms in the $\hat{U}$-momentum equation, is not applicable and leads to a contradiction. 


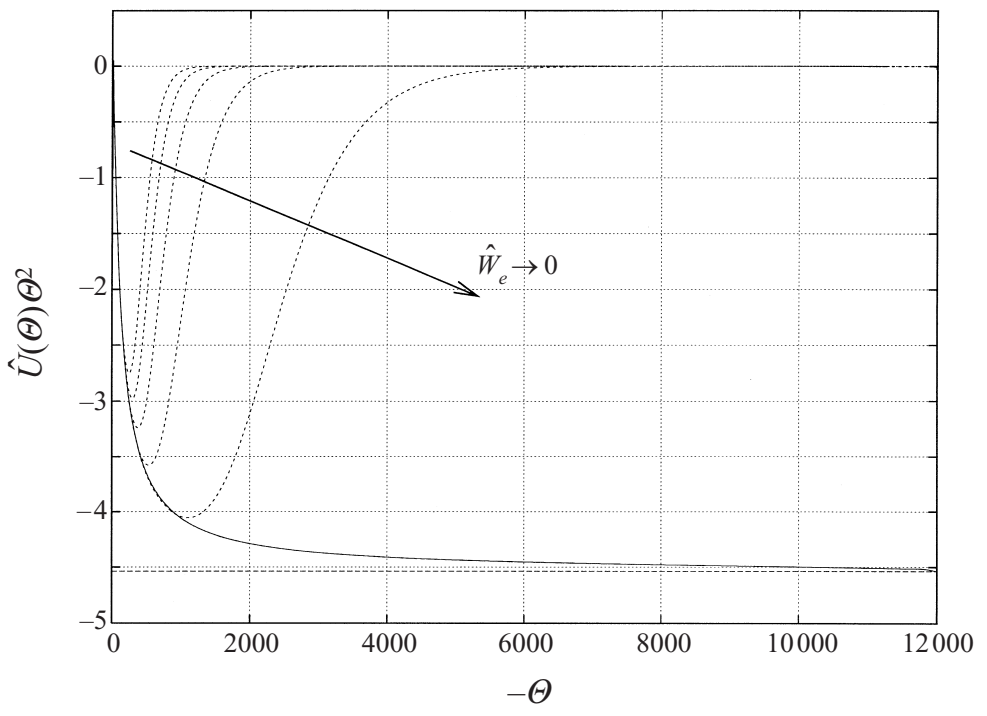

FIgURE 8. A comparison between the predicted, Case (ii), asymptotic solution (solid line) and the full numerical solution (dashed) as $\hat{W}_{e} \rightarrow 0^{-}$, with $S^{*}=0$, and $\sigma=0.5$. $\hat{W}_{e}=-9,-7,-5,-3,-1 \times 10^{-3}$. The horizontal line is at the constant value $U_{0}$ (see the text for a description).

$|\Theta| \gg 1$ behaviour when $\left|\hat{W}_{e}\right| \ll 1$ : Case (iii)

In contrast to the previous two cases there is a large- $|\Theta|$ behaviour that involves an exponential rather than algebraic decay and for which the $\hat{V}$-term approaches a constant at the boundary-layer edge. The appropriate behaviour in this case is

$$
\begin{aligned}
\hat{U} \rightarrow U_{0} \exp \left(\hat{V}_{\infty} \Theta\right), & \hat{V} \rightarrow \hat{V}_{\infty}, \\
B^{*} \rightarrow B_{0} \exp \left(\sigma \hat{V}_{\infty} \Theta\right), & \hat{W} \rightarrow W_{0} \exp \left(\hat{V}_{\infty} \Theta\right),
\end{aligned}
$$

where clearly we require $\hat{V}_{\infty}>0$ and $\sigma>1$ to obtain the necessary balance.

Numerical results for $\left|\hat{W}_{e}\right| \ll 1$

Obtaining solutions to the full boundary-layer equations in the region $\left|\hat{W}_{e}\right| \ll 1$ is numerically difficult because, in general, long lengthscales are required. In figure 8 we show a comparison of the full numerical solution with the predicted asymptotic behaviour for the scaled velocity component $\hat{U}$ (as described by Case (i) above). The numerical results were obtained by following the branch denoted by $(I a)$ in figure 3 but at a Schmidt number of $\sigma=0.5$ as $\hat{W}_{e} \rightarrow 0^{-}$with $S^{*}=\hat{W}_{e}^{2}$. At this value of $\sigma$ the appropriate root of the polynomial (3.12) is $\gamma \approx 1.3491$, leading to $U_{0} \approx-4.5391$, and it is this value that is shown in the figure as the horizontal line.

In figure 9 we show the form of a non-uniform solution branch for $\left|\hat{W}_{e}\right| \ll 1$ (with $\left.S^{*}=W_{e}^{2}\right)$ at a range of Schmidt numbers. Similarly, in figure 10(a) we present a cross-section of the solution surface at fixed $S^{*}=0$ and $\sigma=5.0$. As can be seen from the figure, the structure around $\hat{W}_{e}=0$ is rather complicated. Figure $10(b)$ is a rescaled view of the solution branches shown in figure 10(a). The repeated folding of the solution branch and its intersection with $\hat{W}_{e}=0$ is typical for moderate values of $\sigma$, and the solution branches can be continued further (to lower values of $\hat{V}_{\infty}$ ) but to do so requires very large domains in the computation. The scalings of Case (iii) can be verified at each point where $\hat{W}_{e}=0$ along the solution branches of figure 10 , as shown in figure $11(b)$. Figure $11(a)$ shows profiles of $\hat{V}$ at the points denoted 


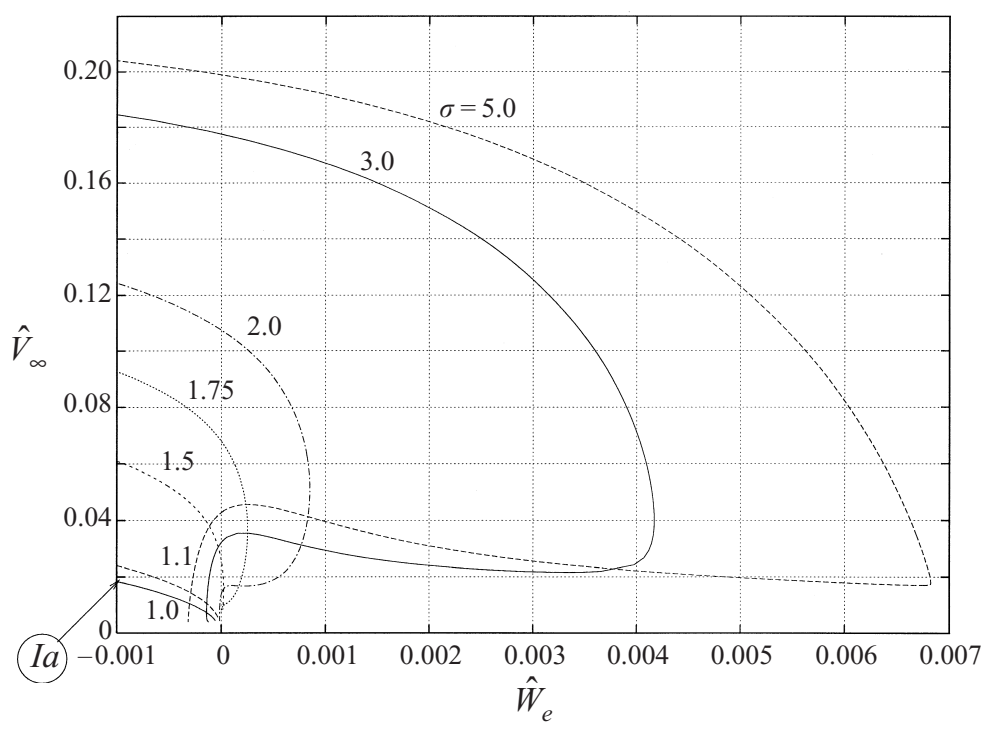

FIGURE 9. Solution branches in the region $\left|\hat{W}_{e}\right| \ll 1$, with $S^{*}=\hat{W}_{e}^{2}$ and varying $\sigma$.

by (i) and (ii) in figure $10(b)$ and (iii) in figure $10(a)$; it is at these points that the comparisons of figure $11(b)$ are made. The resolution of the solution requires a large numerical domain when $\hat{V}_{\infty} \rightarrow 0$ as can be seen from the scalings (3.19).

We must note that the analytical description of the small- $\hat{W}_{e}$ region is not entirely complete. In particular the appropriate form of the solution at large- $|\Theta|$ is unclear when $\sigma \in(40(3-2 \sqrt{2}) / 7 \approx 0.98042,1]$. None of the cases described above are directly applicable in this parameter range; however the numerical results obtained for Schmidt numbers in this region present no convincing evidence that solutions do not exist. Since this region of parameter space is so small it could be that the numerical solutions cannot be continued exactly to $\hat{W}_{e}=S^{*}=0$ but the definitive resolution of this feature is beyond any practical range achievable by the numerical scheme. The alternative is that there is an additional form of large- $|\Theta|$ behaviour that we have not discovered, which is valid within this range of $\sigma$.

\subsection{The region $\hat{W}_{e} \approx 1$}

Linear solutions in the region of parameter space near to $\hat{W}_{e}=1$ correspond to a buoyancy-affected Ekman layer. The linearized analysis of DFH showed that the steady solution presented by Thorpe (1987) was only strictly valid when $\left|\hat{W}_{e}-1\right| \ll E^{2}$, where $E$ is an Ekman number based on the rotation rate of the container. It was also noted by DFH that the preferred final state from the point of view of the readjustment process (with an initial state of rigid body rotation $\hat{W}_{e}=1$ ) was a fully nonlinear solution. The analysis of this nonlinear state near $\hat{W}_{e}=1$ was continued in the later paper by HDDF, in which it was shown that moderate values of $\sigma$ can further complicate the form of the solution branches in this region. The stability analysis of HDDF showed that the primary solution branch was linearly unstable and had both discrete and continuous spectra.

The nonlinear solution available in the region $\hat{W}_{e} \approx 1$ appears as a bifurcation of the trivial state $\hat{W}=B^{*}=1, \hat{U}=\hat{V}=0$ at $\hat{W}_{e}=1, S^{*}=1,1-4 / \sigma$; see figure 12 . It is at this latter value that one of the critical boundaries (denoted by $S_{c r i t}^{*}\left(\hat{W}_{e}, \sigma\right)$, as discussed both by DFH and later in this paper) approaches $\hat{W}_{e}=1$. Near this region 

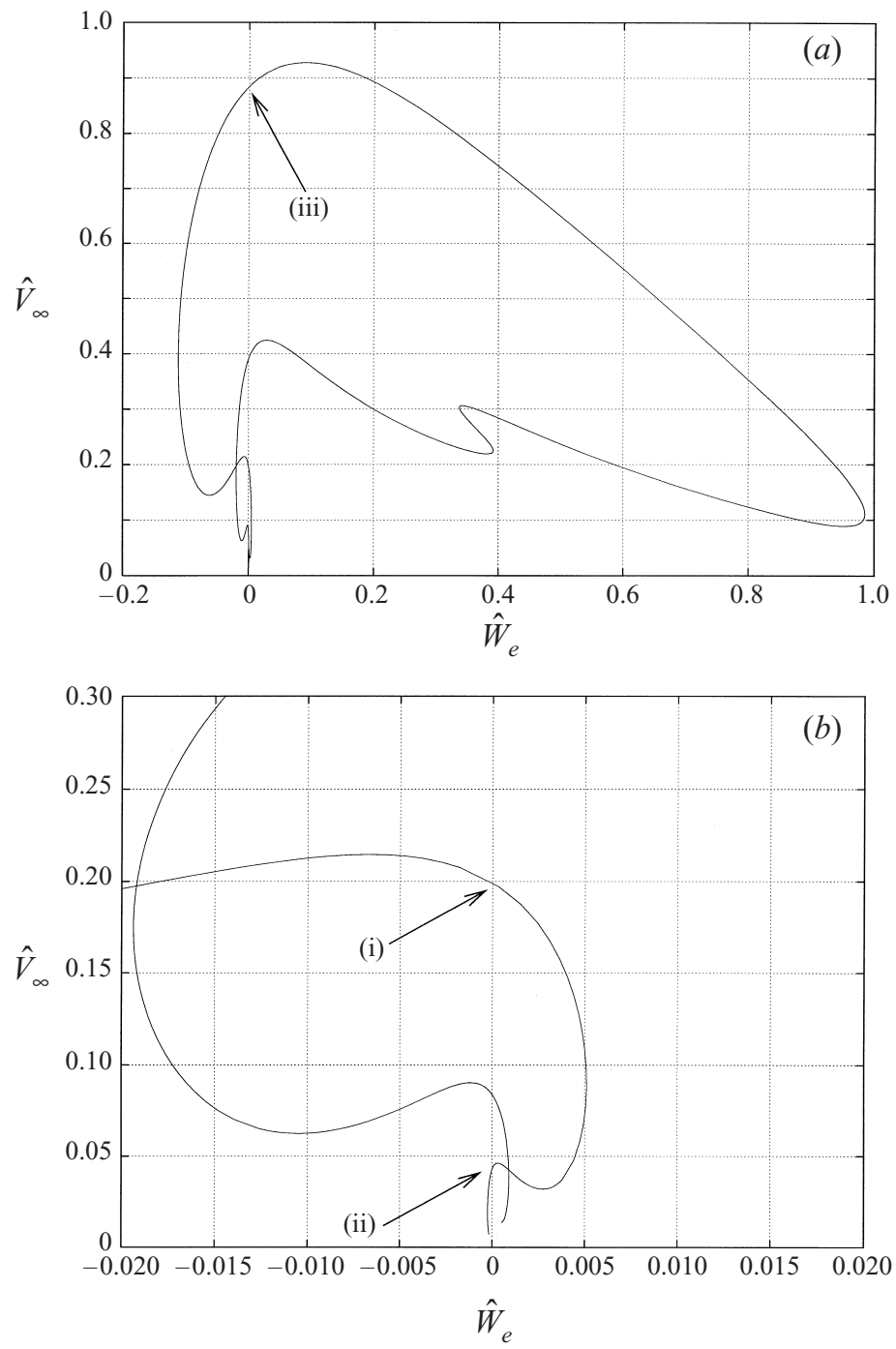

FIGURE 10. A cross-section of a solution surface for fixed $S^{*}=0$ and $\sigma=5 ;(b)$ is an expanded view of the $\left|\hat{W}_{e}\right| \ll 1$ region of $(a)$.

a cusp-catastrophe is introduced into $S^{*}>0$ for sufficiently large Schmidt numbers. In figure 13(a) we show a set of steady states represented as a surface in $\left\{\hat{V}_{\infty}, \hat{W}_{e}, S^{*}\right\}$ space for the case $\sigma=10$. As can be observed from the figure, a fold develops in the locus of $\left\{\hat{V}_{\infty}, \hat{W}_{e}\right\}$ at a critical value of $S^{*}$. The projection of this fold region onto the $\left(S^{*}, \hat{W}_{e}\right)$-plane is (locally) a cusped region, the two boundaries of which are sets of one-sided bifurcation points; see figure 13(b). Viewing the structure of figure 13(a) along the locus in parameter space that bisects the cusp, then the feature would appear as a symmetric bifurcation in which three solutions evolve from one when moving beyond the critical point.

It is worth noting that the behaviour described above is only one example of a feature that can be located at a number of regions in the full parameter space. We should also point out that many of the other bifurcation phenomena described in 

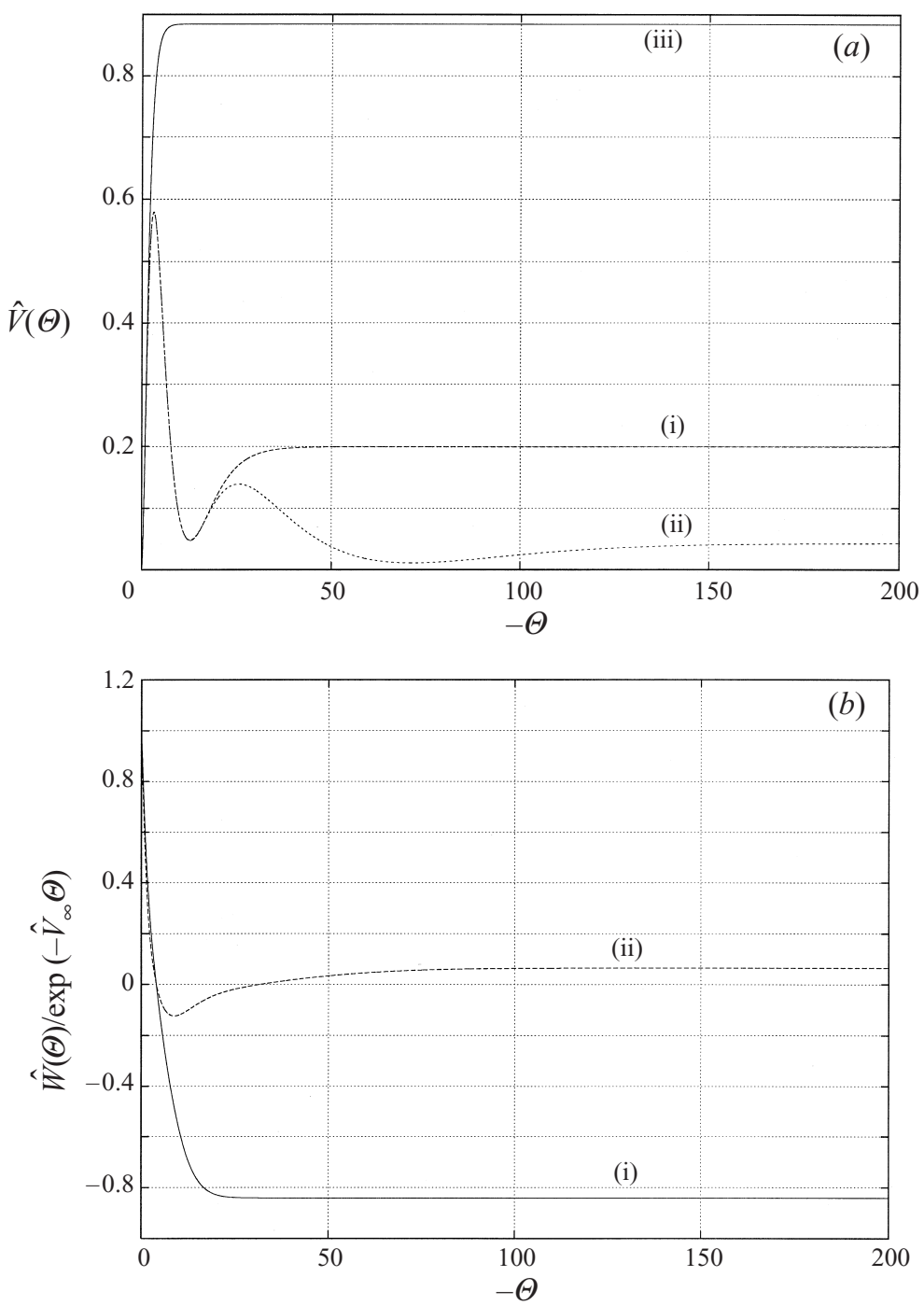

FIGURE 11. The solutions available at $\hat{W}_{e}=S^{*}=0$, with $\sigma=5.0$. (a) Profiles of $\hat{V}(\Theta)$ at points (i), (ii) and (iii) shown in figure 10. (b) Large- $|\Theta|$ decay as described by Case (iii).

this paper have been discussed previously by Benjamin (1978) for general steady flows; nevertheless we find it surprising that so many complex features arise in what appears, at first sight, to be a relatively uncomplicated system.

\subsection{Singular solutions}

It is well known that the RDE have regions in the (one-dimensional) parameter space within which many solutions can be found. In our notation the regions of particular interest are near $\hat{W}_{e}=0$ and $\hat{W}_{e}= \pm 1.4355$. The qualitative behaviour of the region near $\hat{W}_{e}=0$ has been discussed above (figure 2), but when sufficiently close to the other critical values of $\hat{W}_{e}= \pm 1.4355$ it has been shown that one can construct an infinity of solution branches that connect through a singular solution; see Ockendon (1972) and Bodonyi (1973). Ockendon allowed for a small suction applied at the disk 


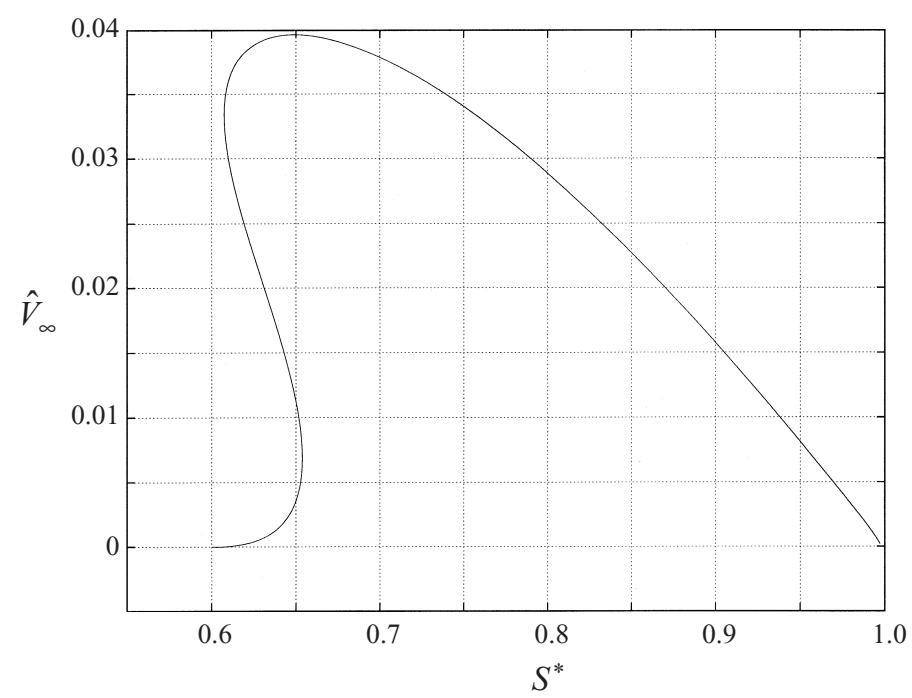

FIGURE 12 . The nonlinear steady state at $\hat{W}_{e}=1$. This state appears as a bifurcation from the trivial state $\left(\hat{U}=0, \hat{V}=0, \hat{W}=1, B^{*}=1\right)$ at $S^{*}=1,1-4 / \sigma$, and is shown here for $\sigma=10$.

and showed that a chain of inviscid cell solutions can be constructed that match each other, the far-field conditions and the boundary conditions at the disk through viscous sublayers. Later, Bodonyi (1975) applied matched asymptotic methods directly to the RDE (without the small suction) to reveal the structure of the solution branches as $\hat{W}_{e} \rightarrow-1.4355$, deducing this critical value directly from the analysis. We note that the solution branches described by the analysis of Bodonyi have $f(z \rightarrow \infty) \rightarrow+\infty$ as $\hat{W}_{e} \rightarrow-1.4355$ (in the notation of (1.7)), and therefore we expect (on considering the form of (2.2)) that the structure may be extended to cover our more general boundary-layer equations (1.1)-(1.4).

In an extension to the analysis of Bodonyi (1975) we shall discuss the form of the primary solution branch as the modified Burger number approaches a limit $S^{*} \rightarrow$ $S_{\text {sing }}^{*}\left(\hat{W}_{e}, \sigma\right)$; this defines a surface in parameter space near which solutions of this asymptotic structure can be located. The exact dependence of $S_{\text {sing }}^{*}$ on the other parameters, $\hat{W}_{e}$ and $\sigma$, will be derived as part of the asymptotic description. By analogy with the work of Bodonyi, we restrict attention to three distinct regions (though higher-branch solutions involving more regions are possible) defined by $\Theta=O\left(\epsilon^{1 / 4}\right), \Theta=O\left(\epsilon^{-1 / 4}\right)$ and $\Theta=-C \epsilon^{-1 / 4}+O\left(\epsilon^{1 / 4}\right)$, where $\epsilon=S^{*}-S_{\text {sing }}^{*}\left(\hat{W}_{e}, \sigma\right)$, $S_{\text {sing }}^{*}$ is to be determined and $C$ is a constant.

\subsubsection{Inner viscous layer: Region I}

In a viscous region adjacent to the boundary we introduce the scaled coordinate $\xi=\Theta \epsilon^{-1 / 4}=O(1)$, and write

$$
\begin{gathered}
\hat{U}=\epsilon^{-2 / 4}\left(U_{0}(\xi)+\epsilon U_{1}(\xi)+\cdots\right), \\
\hat{V}=\epsilon^{-1 / 4}\left(V_{0}(\xi)+\epsilon V_{1}(\xi)+\cdots\right), \\
\hat{W}=W_{0}(\xi)+\epsilon W_{1}(\xi)+\cdots \\
B^{*}=\hat{W}_{e}^{2}+\left(S_{\text {sing }}^{*}-\hat{W}_{e}^{2}\right) B_{0}(\xi)+\epsilon B_{1}(\xi) \cdots .
\end{gathered}
$$



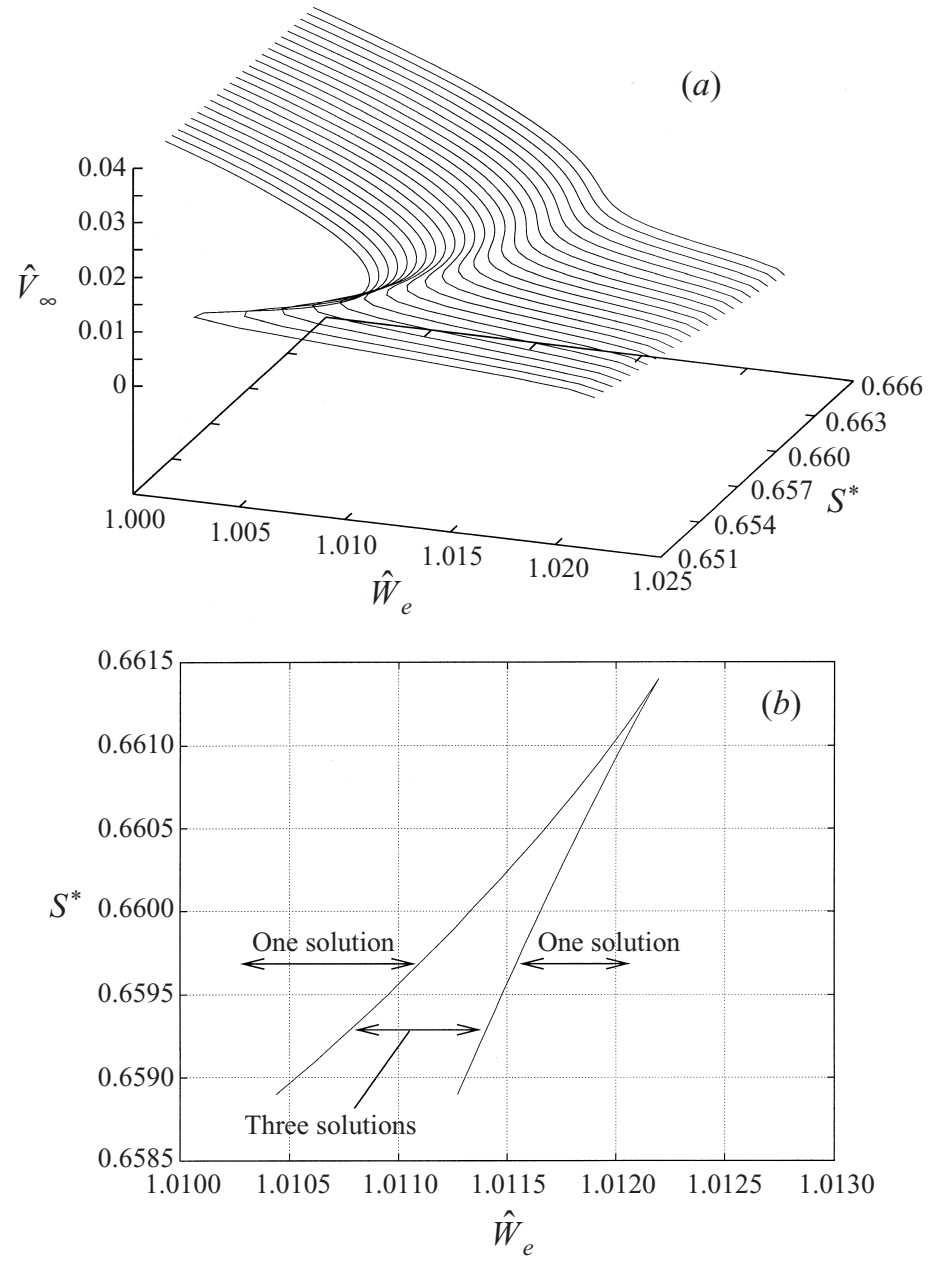

FIGURE 13. Numerical results for steady states near to $\hat{W}_{e}=1, \sigma=10$.

At leading order, after substitution of the above expansions, the governing boundarylayer equations are reduced to

$$
\begin{gathered}
U_{0}^{2}+V_{0} U_{0}^{\prime}=U_{0}^{\prime \prime}, \\
2 U_{0} W_{0}+V_{0} W_{0}^{\prime}=W_{0}^{\prime \prime}, \\
V_{0} B_{0}^{\prime}+U_{0}\left(B_{0}-1\right)=\sigma^{-1} B_{0}^{\prime \prime}, \\
2 U_{0}+V_{0}^{\prime}=0,
\end{gathered}
$$

with boundary conditions $U_{0}=V_{0}=0, W_{0}=1$ on $\xi=0$. There is a simple solution to this system, namely

$$
V_{0}=-A \xi^{2}, \quad U_{0}=A \xi, \quad B_{0}=1 .
$$

At this stage we have used a notation similar to that employed by Bodonyi (1975) (obviously the constant $A$ is unrelated to that used in the preceding sections of this paper); however, there is a sign change in the coordinate since the appropriate domain is $\xi \in[0,-\infty)$ in our notation. Given the above solutions, the equation for 
the azimuthal velocity component, $W_{0}$, reduces to

$$
W_{0}^{\prime \prime}+A \xi^{2} W_{0}^{\prime}-2 A \xi W_{0}=0,
$$

which can be solved in terms of confluent hypergeometric functions, to give

$$
W_{0}=C_{1} \mathscr{U}\left(-\frac{2}{3}, \frac{2}{3},-\frac{A \xi^{3}}{3}\right)+C_{2} \exp \left(-\frac{A \xi^{3}}{3}\right) \mathscr{U}\left(\frac{4}{3}, \frac{2}{3}, \frac{A \xi^{3}}{3}\right) .
$$

Comparison of the shear stress values (at the boundary $\Theta=0$ ) obtained from numerical results indicate that the constant $A$ is positive. Therefore, the large- $\xi$ behaviour together with the no-slip boundary condition determine the constants $C_{1}$ and $C_{2}$ to be

$$
C_{1}=-3 \frac{\Gamma\left(\frac{2}{3}\right)}{\Gamma\left(\frac{1}{3}\right)}, \quad C_{2}=0
$$

Furthermore, as $\xi \rightarrow-\infty$ we have $W_{0} \sim-3^{1 / 3} \Gamma(2 / 3) / \Gamma(1 / 3)\left(-A \xi^{3}\right)^{2 / 3}$, and the expansion fails when $\epsilon W_{0}^{2} / \xi^{2}=O(1)$, suggesting an outer layer needs to be considered.

\subsubsection{Inviscid outer layer: Region II}

This outer region is defined by the scaled coordinate $\eta=\epsilon^{1 / 4} \Theta=O(1)$, and we introduce

$$
\begin{gathered}
\hat{U}=\epsilon^{-1}\left(\bar{U}_{0}(\eta)+\cdots\right), \\
\hat{V}=\epsilon^{-5 / 4}\left(\bar{V}_{0}(\eta)+\cdots\right), \\
\hat{W}=\epsilon^{-1}\left(\bar{W}_{0}(\eta)+\cdots\right), \\
B^{*}=\hat{W}_{e}^{2}+\left(S_{\text {sing }}^{*}-\hat{W}_{e}^{2}\right) \bar{B}_{0}(\eta)+\cdots .
\end{gathered}
$$

Applying these expansions reduces the leading-order system to

$$
\begin{gathered}
\bar{U}_{0}^{2}+\bar{V}_{0} \bar{U}_{0}^{\prime}-\bar{W}_{0}^{2}=0, \\
2 \bar{U}_{0} \bar{W}_{0}+\bar{V}_{0} \bar{W}_{0}^{\prime}=0, \\
\bar{V}_{0} \bar{B}_{0}^{\prime}+\bar{U}_{0}\left(\bar{B}_{0}-1\right)=0, \\
2 \bar{U}_{0}+\bar{V}_{0}^{\prime}=0 .
\end{gathered}
$$

We can give an exact solution to this system,

$$
\begin{gathered}
\bar{U}_{0}=C_{3} \sin \left(C_{5} \eta\right), \quad \bar{V}_{0}=-\frac{2 C_{3}}{C_{5}}\left[1-\cos \left(C_{5} \eta\right)\right], \\
\bar{W}_{0}=C_{3}\left[1-\cos \left(C_{5} \eta\right)\right], \quad \bar{B}_{0}=1+C_{4} \bar{V}_{0}^{1 / 2},
\end{gathered}
$$

where $C_{3}, C_{4}$ and $C_{5}$ are constants. Matching this outer-layer description with the large- $\xi$ form of the solution in region I leads to

$$
C_{3}=\frac{A}{C_{5}}, \quad \text { and } \quad C_{5}=2 \frac{\Gamma\left(\frac{2}{3}\right)}{\Gamma\left(\frac{1}{3}\right)}\left(\frac{3}{A}\right)^{1 / 3} .
$$

We note that $C_{3}$ and $C_{5}$ are both positive, implying $C_{4}=0$ since $\bar{V}_{0}<0$.

\subsubsection{Outer viscous layer: Region III}

The expansion for region II fails when $C_{5} \eta=-2 \pi$, thus we obtain a further viscous outer layer defined by the scaled coordinate $\Theta=-\left(2 \pi / C_{5}\right) \epsilon^{-1 / 4}+\zeta \epsilon^{1 / 4}$. In 
this region the expansions are equivalent to those presented for the inner viscous layer (region I), and the resulting equations are also as given in (3.24)-(3.27). The boundary conditions as $\zeta \rightarrow+\infty$ are determined from the solutions of region II as $\eta \rightarrow 2 \pi$. As noted by Bodonyi, there are two possible outer boundary conditions for this region, corresponding to matching with the far-field solution $\left(\hat{U} \rightarrow 0, \hat{W} \rightarrow \hat{W}_{e}\right.$, $B^{*} \rightarrow \hat{W}_{e}^{2}$ ) or matching with a further inviscid outer region of the form described above. In this way an arbitrarily large number of solution branches can be obtained.

We note that at this order the constant $A$ is undetermined. For comparisons between the asymptotic description and the numerical results it is sufficient to compute an approximation to $A$ by considering the shear stress components as $S^{*} \rightarrow S_{\text {sing }}^{*}$.

\subsubsection{The critical boundary $S^{*}=S_{\text {sing }}^{*}\left(\hat{W}_{e}, \sigma\right)$}

We can determine the critical value of $S^{*}$ (given $\hat{W}_{e}$ and $\sigma$ ) by continuing the analysis of region I to next order. In particular, substitution of the relevant expansions into the $\hat{U}$-momentum equation leads to

$$
V_{1}^{\prime \prime \prime}+A \xi^{2} V_{1}^{\prime \prime}-2 A \xi V_{1}^{\prime}+2 A V_{1}=2\left(W_{0}^{2}-S_{\text {sing }}^{*}\right) .
$$

Equation (3.43) has been considered by Bodonyi (1973), and we derive a similar solution to this system with little alteration:

$$
V_{1}(\xi)=C_{6} \xi^{2}+2 \xi \int_{0}^{-\xi} \int_{0}^{\gamma} \exp \left(\frac{A \eta^{3}}{3}\right) \int_{0}^{\eta} x^{2}\left(W_{0}^{2}-S_{\text {sing }}^{*}\right) \exp \left(-\frac{A x^{3}}{3}\right) \mathrm{d} x \mathrm{~d} \eta \mathrm{d} \gamma,
$$

where $C_{6}$ is a constant of integration. Differentiation of this expression leads to

$$
\frac{V_{1}^{\prime \prime \prime}(\xi)}{2}=W_{0}^{2}(\xi)-\exp \left(-\frac{A \xi^{3}}{3}\right)\left\{S_{\text {sing }}^{*}-A \int_{0}^{-\xi} x^{2} W_{0}^{2}(x) \exp \left(-\frac{A x^{3}}{3}\right) \mathrm{d} x\right\} ;
$$

therefore, since $A>0$ and $\xi \in[0,-\infty)$, eliminating the exponentially growing term in (3.45) provides a surprisingly simple extension to the result of Bodonyi (1975), namely

$$
S_{\text {sing }}^{*}=\frac{9 \Gamma^{2}\left(\frac{2}{3}\right)}{\Gamma^{2}\left(\frac{1}{3}\right)} \int_{0}^{\infty} \mathscr{U}\left(-\frac{2}{3}, \frac{2}{3}, t\right) \exp (-t) \mathrm{d} t .
$$

The expression (3.46) shows that, in terms of the $S^{*}, \hat{W}_{e}$ parameter space, the singular solutions are available along a line of constant $S^{*}=S_{\text {sing }}^{*} \approx 2.061$; that is, the critical value is independent of both $\hat{W}_{e}$ and the Schmidt number $\sigma$. Numerical results showing the behaviour of $\hat{V}_{\infty}$ as the critical value of $S^{*}$ is approached are given in figure 14. The values of $\hat{W}_{e}$ and $\sigma$ were arbitrarily chosen to demonstrate that $S_{\text {sing }}^{*}$ is indeed independent of the other parameters.

It is also interesting to note the form of the profiles for $B^{*}$, which are uniform over the majority of the boundary layer. In regions I and II, $B^{*}=S_{\text {sing }}^{*}+O(\epsilon)$, with a rapid transition to the edge conditions $\left(B^{*}=\hat{W}_{e}^{2}\right)$ in a thin, $O\left(\epsilon^{1 / 4}\right)$, outer viscous layer. Figure 15 shows profiles of $\hat{W}$ and $B^{*}$ as $S^{*} \rightarrow S_{\text {sing }}^{*}$; the outer viscous region (III) is clearly seen in the otherwise uniform $B^{*}$-profiles.

As we have already noted, the solutions to the RDE are applicable to the more general boundary-layer system along the parabola $S^{*}=\hat{W}_{e}^{2}$. This parabola intersects $S^{*}=S_{\text {sing }}^{*}$ at $\hat{W}_{e}= \pm 1.4355$, at which points the $B^{*}$-profiles are uniform throughout the whole domain and the above expansion reduces to the form given by Bodonyi (1975). 

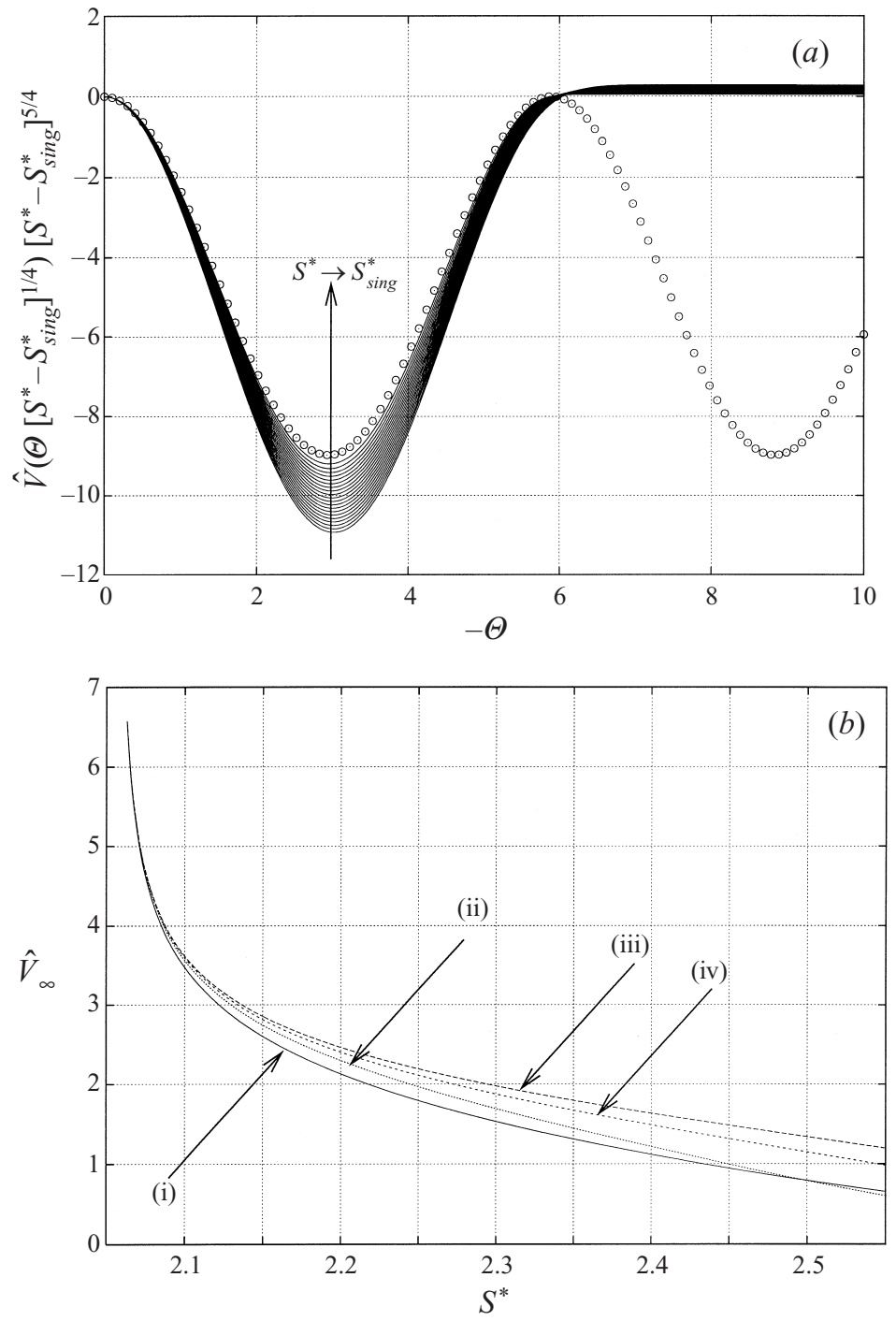

FIGURE 14. The singular solution branch as $S^{*} \rightarrow S_{\text {sing. }}^{*}$ (a) A comparison between scaled numerical results (solid lines) and the asymptotic result for region II (shown as data points for clarity) as $S^{*} \rightarrow S_{\text {sing. }}^{*}$ (b) The behaviour of $\hat{V}_{\infty}$ for varying $S^{*}:$ (i) $\hat{W}_{e}=-1.4, \sigma=5$. (ii) $\hat{W}_{e}=-0.5, \sigma=1$. (iii) $\hat{W}_{e}=-0.5, \sigma=5$. (iv) $\hat{W}_{e}=0.5, \sigma=5$.

\subsection{The limit $\hat{V}_{\infty} \rightarrow 0$}

DFH considered the readjustment of a linearly stratified fluid (in a conical container) to an impulsively imposed change in the container rotation rate. The final result of their analysis was that, at order-one values of the Schmidt number, the evolution could be classified into three main types; the effect of higher values of $\sigma$ was later considered by HDDF. The boundaries that separate the evolution types in parameter space were shown to be either explicitly known, obtained (numerically) from a reduced asymptotic problem or determined by a stability analysis. Specifically, the boundary that separated parameter values for which a steady state could be achieved from a scenario involving a gradual thickening of the boundary layer was determined by 

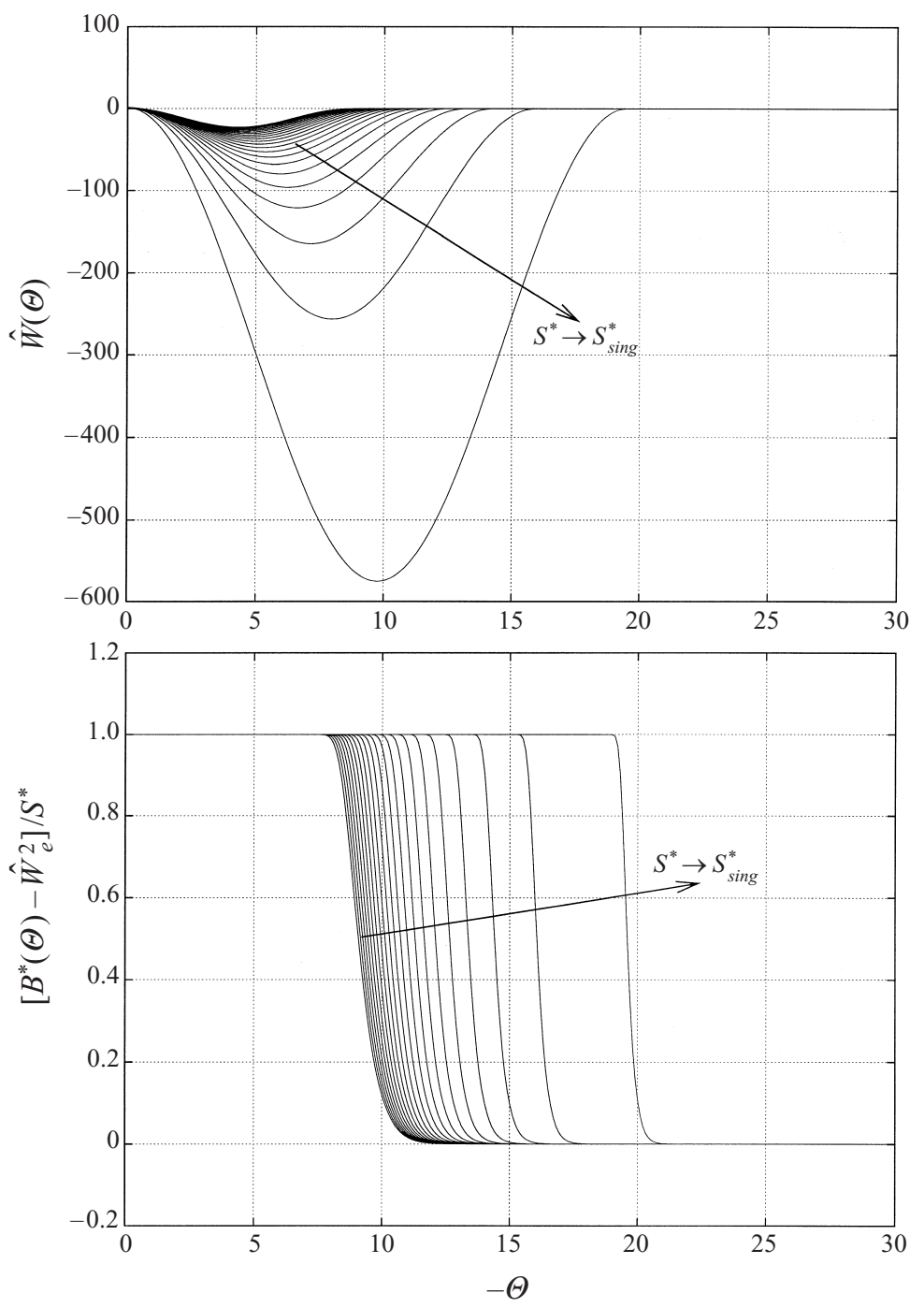

Figure 15. Profiles of $\hat{W}$ and $\left(B^{*}-\hat{W}_{e}^{2}\right) / S^{*}$ as $S^{*} \rightarrow S_{\text {sing }}^{*} ; S^{*} \in[2.299,2.068]$, $\hat{W}_{e}=-0.5$ and $\sigma=1$.

considering steady solutions with $0<\hat{V}_{\infty} \ll 1$. The growing boundary-layer regime was found to be a preferred state only for parameter values to which the steady state could not be extended, that is, for $S^{*}>S_{\text {crit }}^{*}$ (in the DFH notation). Therefore, boundaries beyond which solutions cannot be continued can be of some practical importance both when considering time-dependent readjustment processes and when choosing parameter values for experimental work.

The description of the boundary $S_{c r i t}^{*}$ is as follows. For a small perturbation about the critical value of the form

$$
S^{*}=S_{c r i t}^{*}+\delta
$$

we introduce the following expansion in a $\Theta=O(1)$ layer adjacent to the $\Theta=0$ 
boundary:

$$
\left\{\hat{U}, \hat{V}, \hat{W}, B^{*}\right\}^{T}=\{0,0,1,1\}^{T}+\delta\left\{U_{1}(\Theta), V_{1}(\Theta), W_{1}(\Theta), B_{1}(\Theta)\right\}^{T}+\cdots .
$$

Substitution into the governing boundary-layer equations leads to the following system for $\left\{U_{1}, V_{1}, W_{1}, B_{1}\right\}^{T}$ :

$$
\begin{gathered}
-2 W_{1}=U_{1}^{\prime \prime}-B_{1}, \\
2 U_{1}=W_{1}^{\prime \prime}, \\
U_{1}\left(1-S_{c r i t}^{*}\right)=\frac{1}{\sigma} B_{1}^{\prime \prime}, \\
2 U_{1}+V_{1}^{\prime}=0,
\end{gathered}
$$

with conditions $U_{1}(0)=V_{1}(0)=B_{1}^{\prime}(0)=0$ and $W_{1}(0)=1$ on $\Theta=0$. Solution of (3.49)-(3.52) leads to

$$
\begin{gathered}
V_{1}=-2 C\left\{\frac{E^{+}}{\lambda(1+\mathrm{i})}-\frac{E^{-}}{\lambda(1-\mathrm{i})}\right\}-\frac{2 \mathrm{iC}}{\lambda}, \\
W_{1}=\mathrm{i} C\left\{\frac{2}{\lambda^{2}}-\frac{1}{\lambda^{2}}\left(E^{+}+E^{-}\right)-2 \lambda \Theta\left(\lambda^{2}-\frac{1}{\lambda^{2}}\right)\right\}, \\
B_{1}=\mathrm{i} C\left\{2\left(E^{+}+E^{-}\right)\left[\lambda^{2}-\frac{1}{\lambda^{2}}\right]-4 \lambda \Theta\left[\lambda^{2}-\frac{1}{\lambda^{2}}\right]+\frac{4}{\lambda^{2}}\right\},
\end{gathered}
$$

where $C$ is a constant, $E^{ \pm} \equiv \exp [\lambda(1 \pm \mathrm{i}) \Theta]$, and

$$
\lambda^{4}=\frac{1}{4}\left[4-\sigma\left(1-S_{c r i t}^{*}\right)\right] .
$$

For $\Theta \rightarrow-\infty$ we observe that

$$
U_{1} \rightarrow 0, \quad V_{1} \rightarrow \mu, \quad W_{1} \sim \mu \Theta\left(\lambda^{4}-1\right),
$$

where $\mu=-2 \mathrm{i} C / \lambda$. As we noted in $\S 3.2, S^{*}=1-4 / \sigma$ is a limiting value and corresponds to $\lambda \rightarrow 0$ in the above description.

The large $-|\Theta|$ behaviour in this inner solution, together with the form of the expansions (3.48) suggests an outer layer defined in terms of the scaled coordinate $\tilde{\Theta}=\delta \Theta=O(1)$, in which the appropriate expansion is

$$
\begin{aligned}
&\left\{\hat{U}, \hat{V}, \hat{W}, B^{*}\right\}^{T}=\left\{\delta^{2} \tilde{U}_{1}(\tilde{\Theta})+\cdots, \delta \tilde{V}_{1}(\tilde{\Theta})+\cdots\right. \\
&\left.\tilde{W}_{1}(\tilde{\Theta})+\cdots, \tilde{B}_{1}(\tilde{\Theta})+\cdots\right\}^{T}+\cdots .
\end{aligned}
$$

The equations governing the leading-order solution in this outer layer are

$$
\begin{gathered}
\tilde{W}_{1}^{2}=\tilde{B}_{1}, \\
2 \tilde{U}_{1} \tilde{W}_{1}+\tilde{V}_{1} \tilde{W}_{1}^{\prime}=\tilde{W}_{1}^{\prime \prime}, \\
\tilde{V}_{1} \tilde{B}_{1}^{\prime}+\tilde{U}_{1}\left(\tilde{B}_{1}-S_{c r i t}^{*}\right)=\frac{1}{\sigma} \tilde{B}_{1}^{\prime \prime}, \\
2 \tilde{U}_{1}+\tilde{V}_{1}^{\prime}=0 .
\end{gathered}
$$

This system has to be solved according to the conditions (3.57) at $\tilde{\Theta}=0$ and $\tilde{U}_{1} \rightarrow 0$, $\tilde{W}_{1} \rightarrow \hat{W}_{e}$ and $\tilde{B}_{1} \rightarrow \hat{W}_{e}^{2}$ as $[\operatorname{sign}(\delta) \tilde{\Theta}] \rightarrow-\infty$.

A subtlety of this expansion is that the constant $\mu$ can be set to unity by a suitable redefinition of the quantities $\tilde{V}_{1}$ and $\tilde{\Theta}$ in the outer-layer description; however, the sign of $\mu$ remains important when determining the appropriate solution domain. It is 


$$
\begin{array}{lll}
\text { Branch-1: } & w_{0}=1.0 & w_{1}=-0.1542 \\
\text { Branch-2: } & w_{2}=-0.0983 & w_{3}=0.0571 \\
\text { Branch-3: } & w_{4}=0.0565 & w_{5}=-0.0442
\end{array}
$$

TABLE 2. Some of the values $\hat{W}_{e}=w_{i}$ for which rotating disk solutions exist with zero normal velocity far from the disk.

only by comparing the above description with a numerical solution of the full system that the sign of $\mu$ can be obtained and it is this that fixes the appropriate sign for $\delta$ (in order to maintain a solution domain for which the outer solution is exponentially decaying rather than growing). It is in this way that we can determine on which 'side' of the $S_{\text {crit }}^{*}$ boundary steady states can be located.

The outer-layer system (on setting $\mu= \pm 1$ as appropriate) can be combined into a single third-order system and solved by the application of a simple Runge-Kutta method. Iterating on the value of $S_{c r i t}^{*}$ for a given $\hat{W}_{e}$ enables the edge conditions to be satisfied; thus we obtain $S_{c r i t}^{*}\left(\hat{W}_{e}, \sigma\right)$. For a sequence of numerical results concerning $S_{c r i t}^{*}\left(\hat{W}_{e}, \sigma\right)$ the reader is referred to the paper of HDDF.

Given the wide range of solutions that are available to the full governing boundarylayer equations and the level of non-uniqueness it would be surprising if the above analysis was the only $\left|\hat{V}_{\infty}\right| \ll 1$ description. In fact, other expansions that lead to different $\left(S_{\text {crit }}^{*}\right)$ boundaries in the $\left(S^{*}, \hat{W}_{e}\right)$ parameter space are possible.

The crucial feature of the expansion (3.48) is that, for the inner layer, $\hat{V}=O(\delta)$ as $\Theta \rightarrow-\infty$. In the case discussed above this is achieved because the leading-order solution (3.48) in the inner layer is simply the trivial solution $(\hat{U}=\hat{V}=0, \hat{W}=$ $\left.B^{*}=1\right)$. This $\left|\hat{V}_{\infty}\right| \ll 1$ description is non-unique since, rather than the expansion (3.48), we can introduce a more general inner-layer expansion,

$$
\left\{\hat{U}, \hat{V}, \hat{W}, B^{*}\right\}^{T}=\left\{U_{0}(\Theta), V_{0}(\Theta), W_{0}(\Theta), w_{i}^{2}\right\}^{T}+O(\delta),
$$

where $\left\{U_{0}, V_{0}, W_{0}\right\}$ is a solution of the rotating disk equations with $W_{0} \rightarrow w_{i}$ as $\Theta \rightarrow-\infty, B^{*}=w_{i}^{2}$ a constant, and $w_{i}$ is such that $V_{0} \rightarrow 0$ as $\Theta \rightarrow-\infty$. It is well known that there are an infinity of solutions to the rotating disk equations that have zero axial flow; some typical values are given in table 2 (also see figure 2)

In this more general description, the critical value of the modified Burger number is fixed to be $S_{c r i t}^{*}=w_{i}^{2}$ for consistency of the inner-layer description. However, we note that when $i=0$ the corresponding rotating disk solution is the trivial state and in this case the critical value of $S^{*}$ remains undetermined until the next-order inner layer is considered and matched to a corresponding outer layer. In this case the derivation reverts to that summarized above (3.47)-(3.62). For $i \neq 0$ the critical value $S_{c r i t}^{*}=w_{i}^{2}$ is independent of $\hat{W}_{e}$; the outer layer in this case has the same scalings noted above (3.58) and acts to adjust the solution to the appropriate edge behaviour.

In figure 16 we show some numerical results, obtained for the full governing equations (1.1)-(1.6), for solution branches at $\hat{W}_{e}=-0.2,-0.5$ and $\sigma=1,5$. Figure $16(a)$ shows the behaviour of $\hat{V}_{\infty}$ as $S^{*}$ is decreased towards the value $S^{*}=w_{1}^{2}$ (given in table 2); as predicted, $\hat{V}_{\infty}$ approaches zero as $S^{*} \rightarrow w_{1}^{2} \approx 0.02378$. Figure $16(b)$ shows the behaviour of the stress component $\hat{U}^{\prime}(0)$ at the disk and, in agreement with the above inner-layer description for $S^{*} \rightarrow w_{1}^{2}$, we can observe that the values approach that associated with the appropriate rotating disk solution, as displayed by the horizontal line. Figure 17 shows profiles of the normal velocity component $\hat{V}(\Theta)$ 

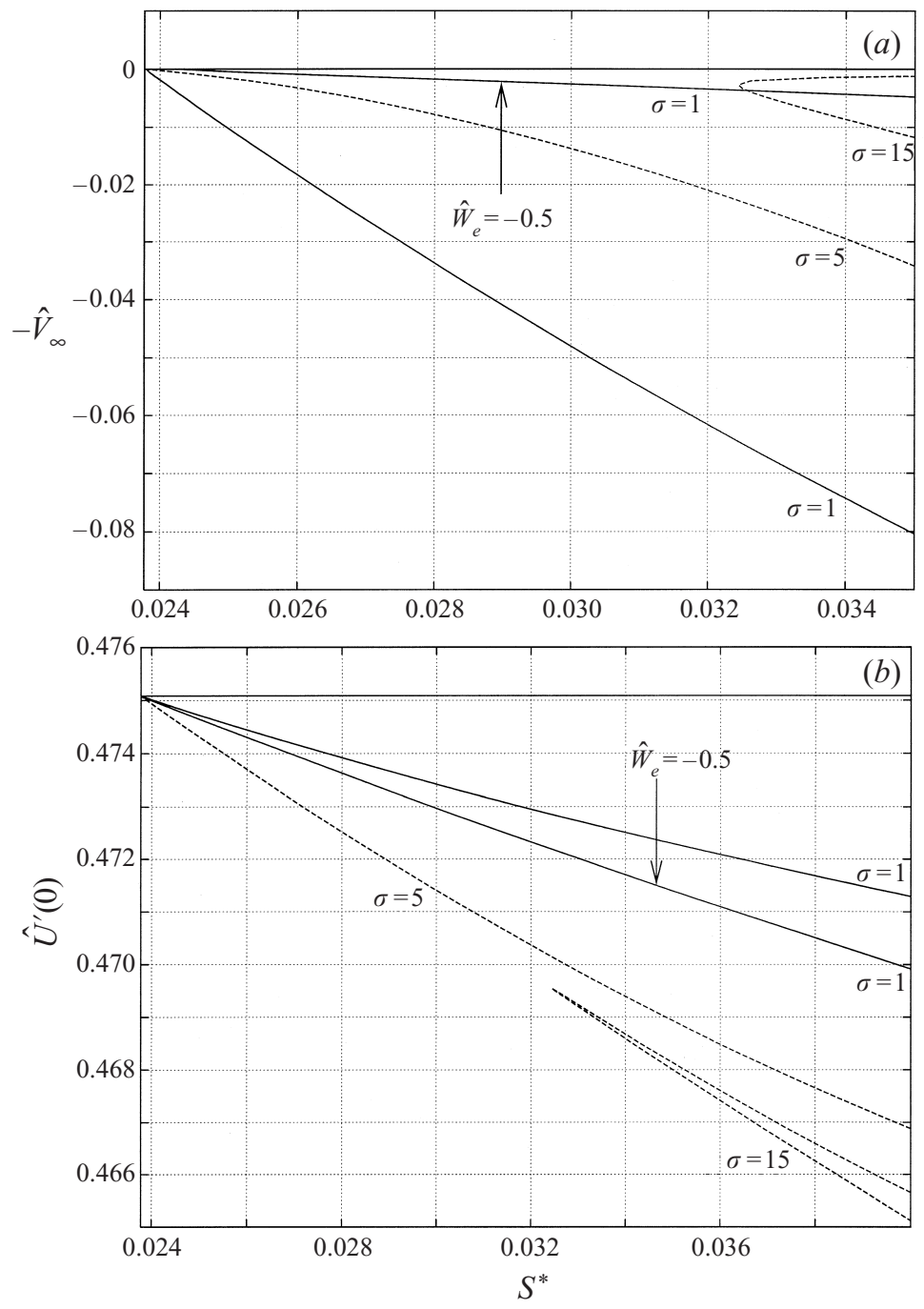

FIGURE 16. Comparison between asymptotic predictions and numerical results; $\hat{W}_{e}=-0.2$ unless otherwise noted.

(over an $O(1)$ inner-layer scale) as $S^{*} \rightarrow w_{1}^{2} \approx 0.02378$ for $\sigma=1$. The figure clearly shows the profiles approaching the limiting form of the rotating disk solution (shown as data points for clarity).

In figure $16(a, b)$ we have also shown numerical results for $\hat{W}_{e}=-0.2$ and $\sigma=15$; in this case the steady solution does not approach $\hat{V}_{\infty}=0$ at $S^{*}=w_{1}^{2}$ since there is a fold in the solution at a larger value of $S^{*}$. This must be the case at these parameter values since examination of the outer-layer equations (3.58), (3.59)-(3.62) reveals that

$$
\begin{aligned}
\tilde{W}_{1} & \sim \hat{W}_{e}+\tilde{w}_{1} \exp \left(c_{1} \tilde{\Theta}\right), \\
\tilde{V}_{1} & \sim \tilde{V}_{e}+\tilde{v}_{1} \exp \left(c_{1} \tilde{\Theta}\right)
\end{aligned}
$$




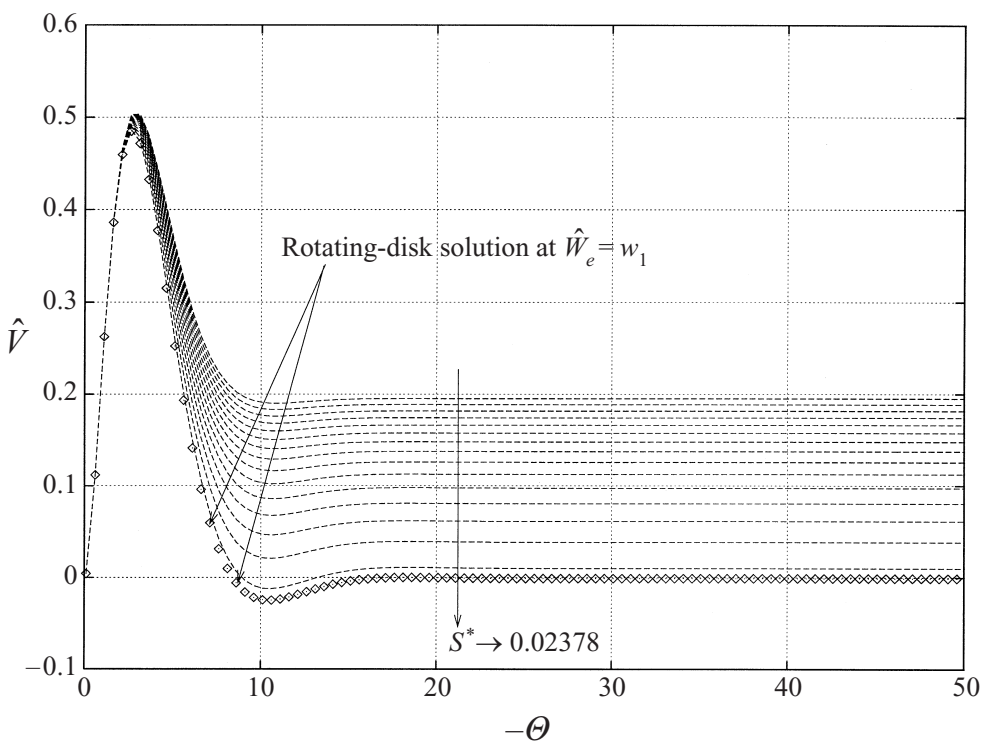

FIGURE 17. $\hat{V}$-profiles for $\hat{W}_{e}=-0.17$ as $S^{*} \rightarrow S_{1}^{*}$ compared to the inner-layer asymptotic solution.

as $\tilde{\Theta} \rightarrow-\infty$, where

$$
c_{1}=\sigma \tilde{V}_{e}\left(\frac{3 \hat{W}_{e}^{2}+S^{*}}{4 \hat{W}_{e}^{2}-\sigma\left(\hat{W}_{e}^{2}-S^{*}\right)}\right) .
$$

Therefore, there is a critical value of $\sigma=\sigma_{\text {crit }} \equiv 4 \hat{W}_{e}^{2} /\left(\hat{W}_{e}^{2}-S^{*}\right)$, for $\tilde{V}_{e}>0$, $S^{*}<\hat{W}_{e}^{2}$, above which an unacceptable exponential growth is obtained in the outerlayer equations. We see that when $\hat{W}_{e}=-0.2, S^{*}=w_{1}^{2} \approx 0.02378$ we should expect to find no solutions with $0<\hat{V}_{\infty} \ll 1$ for $\sigma>\sigma_{c r i t} \approx 10$, which is in agreement with the results of figure 11. For $\sigma=15$ the solution branch folds and can eventually be described by the above $\left|\hat{V}_{\infty}\right| \ll 1$ description, but at a higher critical $S^{*}$ for which exponential decay can be achieved in the outer-layer equations.

We have already noted that the critical boundary that is dependent on $\hat{W}_{e}$ (near which the inner-layer behaviour is the trivial rotating disk solution) figures prominently in the parameter-space diagrams used to interpret the unsteady spin-up readjustment as presented by DFH and later confirmed experimentally by HDDF. It could perhaps be argued that this is the case because it is the relevant boundary for the dominant stable steady state. If this is so, then the additional boundaries at $S^{*}=w_{i}^{2}$ as provided by a more general description of the $\left|\hat{V}_{\infty}\right| \ll 1$ limit may be associated only with states that are unstable and therefore of limited relevance to the large-time unsteady evolution of spin-up/down flows. Such questions cannot be addressed without a detailed stability analysis.

\section{Discussion}

We have considered axisymmetric steady states available within the boundary layer on the inside of a rotating, conical container of swirling, linearly stratified fluid. We have described how the steady states depend on the physical parameters governing the relative rotation, strength of stratification and diffusivity properties of the fluid and 
have shown a remarkably complicated behaviour. Although the description of such states has some inherent interest, our motivation for considering the system in such detail is the hope that a full understanding of the steady phenomena will assist in a continued theoretical/experimental analysis of the unsteady, nonlinear readjustment mechanisms in spin-up (or spin-down, spin-over) problems. This analysis is also the first step in a discussion of the spin-over parameter regime $\left(\hat{W}_{e}<0\right)$.

We have presented a general analysis of the steady states through a combination of numerical continuation methods and bifurcation analysis, together with asymptotic descriptions where possible. At general points within the parameter space we have shown that it is possible to locate a large number of steady states, and that continuation of these states can be complicated unless numerical approaches that can effectively deal with repeated folding of the solution branch are utilized.

The stability of all the states described herein remains an open question. However, in a recent experimental/theoretical investigation into spin-up of a stratified fluid in a cone (HDDF), some of the non-unique steady states described in this work were obtained numerically at parameter values that were open to laboratory investigation. A numerical linear stability analysis of these states (though not exhaustive) suggested that there is either none or at most one stable state in the region of parameter space $\hat{W}_{e} \geqslant 0, S^{*} \geqslant \hat{W}_{e}^{2}$. (Note that the higher-branch states of the rotating-disk equations were shown to be unstable by Bodonyi \& Ng 1984.) We have also shown that the structure of the singular solutions to the rotating-disk equations can be extended to provide similar solutions to the boundary-layer system we consider here. It is known for the rotating-disk equations that the steady states become increasingly (linearly) unstable as $\hat{W}_{e}$ is varied so that the singular solution is approached and we expect a similar result can be obtained here. At parameter values that are less accessible to laboratory experiments, HDDF showed that the stability analysis can be extremely complicated with the eigenvalue spectrum arising from a linear stability analysis having both discrete and continuous components which may lead to instability. We should note that when referring to a state as linearly 'stable' we mean within the framework of the similarity equations; there is no reason to assume that the boundary-layer states are also stable to more general forms of disturbance.

Stability issues in the half-plane of parameter space $\hat{W}_{e}<0$ may be equally complicated to address. Of particular interest in this case is that the unsteady boundary-layer equations can show an evolution to a finite-time breakdown. It is of interest to determine if this unsteady breakdown can be related to the absence of steady states, or an instability of steady states.

The equations governing the swirling flow above a rotating disk are seen to be a sub-class of the boundary-layer equations we have considered here. (Although for the more general geometry the states are no longer exact solutions to the Navier-Stokes system.) This has allowed us to use the well known solutions to the rotating disk equations as a starting point for our more general boundary-layer system. Indeed, many of the problems obtained by considering limiting cases have some relationship to the rotating disk solutions.

Perhaps most importantly, we have shown that there is a range of steady states to the stratified problem that can be traced back to the bifurcation of a (stratified boundary-layer) solution branch from the rotating disk solution near $\hat{W}_{e} \approx-0.1487$ (for $\sigma=1$ ). The presence of this branch ensures that steady states can be found at general points in $\hat{W}_{e}<0, S^{*}>\hat{W}_{e}^{2}$. The local analysis of this imperfect bifurcation also provides details of the fold structure of neighbouring solutions.

In the Appendix we give details of a closely related analysis showing the existence 
of an exact solution to the Navier-Stokes equation, which appears as the bifurcation of a non-axisymmetric state from a rotating disk solution of von Kármán form. In fact the similarity form of von Kármán is preserved for this new branch. The presence of these non-axisymmetric states is prompting future work in the area of non-axisymmetric boundary-layer solutions for a stratified fluid in a rotating cone. The presence of non-axisymmetric flow during the transient response of a rotating stratified fluid in a conical container has been reported previously in the experiments of HDDF.

We should note that the details of an extension of the non-axisymmetric analysis to the more complex boundary-layer equations we consider in the main body of this paper are not trivial. In particular, we can consider the boundary layer on the interior of a conical container filled with homogeneous fluid, which leads to the same governing equations as those in the Appendix (A 5)-(A 7) but with the transformation

$$
\frac{\partial}{\partial \phi} \rightarrow \frac{1}{\cos \alpha} \frac{\partial}{\partial \phi},
$$

where $\alpha$ is the angle of the cone walls to the 'horizontal'. As an immediate consequence the bifurcation at $\hat{W}_{e}=W_{\text {bif }} \approx-0.14485$ discussed in the Appendix is only present when $n / \cos \alpha=2$, where $n$ is an azimuthal wavenumber. Thus there is a bifurcation to the $n=2$ solution for the flat-disk case $(\alpha=0)$ and a similar bifurcation to an $n=1$ mode when $\cos \alpha=1 / 2$. In fact, we have been unable to continue a generalization of the non-axisymmetric solution to non-zero $\alpha$ in this problem. Nevertheless, the existence of steady non-axisymmetric states, or even similar periodic states for a conical/stratified flow configuration remains an open question, and is the subject of ongoing work.

The support of the EPSRC is gratefully acknowledged.

\section{Appendix. A bifurcation to non-axisymmetric von Kármán flows}

An exact solution to the Navier-Stokes equations corresponding to the swirling flow above a differentially rotating disk has been known to exist for some time. Von Kármán (1921) first introduced the appropriate similarity form in the case of a rotating plane in a stationary fluid.

We utilize a cylindrical polar coordinate system $(r, \phi, z)$ centred on the axis of rotation of an infinite disk in a swirling fluid, with associated velocity components $(U, W, V)$ and pressure $P$. A solution of the resulting governing system can be sought with a radial dependence of the form first presented by von Kármán, while also maintaining the dependence on the azimuthal coordinate $\phi$ :

$$
\begin{gathered}
(U, V, W)^{T}=\left(r \hat{U}(\eta, \phi), E^{1 / 2} \hat{V}(\eta, \phi), r \hat{W}(\eta, \phi)\right)^{T}, \\
P=r^{2} \hat{W}_{e}^{2} / 2+E Q(\eta, \phi) .
\end{gathered}
$$

Here $E=v /\left(\Omega h^{2}\right)$ is the Ekman number and $\eta=E^{1 / 2} z$ is a boundary-layer coordinate. (We have tried to retain much of our previous notation, with $\eta$ negative and $\hat{W}_{e}$ denoting the ratio of the angular frequency of far-field fluid and disk.) The boundary conditions are azimuthal periodicity, together with

$$
\hat{U}=\hat{V}=0, \hat{W}=1 \quad \text { on } \quad \eta=0,
$$


and

$$
\hat{U} \rightarrow 0, \hat{W} \rightarrow \hat{W}_{e} \quad \text { as } \quad \eta \rightarrow-\infty,
$$

which are the usual axisymmetric boundary conditions for rotating disk flow.

For this general non-axisymmetric flow the Navier-Stokes equations can be reduced under a boundary-layer approximation to the fifth-order system

$$
\begin{gathered}
\hat{U}^{2}+\hat{V} \hat{U}_{\eta}-\hat{W}^{2}+\hat{W} \hat{U}_{\phi}=\hat{U}_{\eta \eta}-\hat{W}_{e}^{2}, \\
2 \hat{U} \hat{W}+\hat{V} \hat{W}_{\eta}+\hat{W} \hat{W}_{\phi}=\hat{W}_{\eta \eta}, \\
2 \hat{U}+\hat{V}_{\eta}+\hat{W}_{\phi}=0 .
\end{gathered}
$$

These are the boundary-layer equations relevant to a non-axisymmetric flow above a rotating disk. It is well known that the axisymmetric solutions to (A 5)-(A 7) also form an exact solution to the Navier-Stokes equations; that is, although the solution is of boundary-layer form there is no approximation. It will be shown below that there is also a class of non-axisymmetric solution that form an exact solution.

The general system (A 5)-(A 7) was investigated by looking for a solution of the form

$$
(\hat{U}, \hat{V}, \hat{W})^{T}=\left(U_{0}(\eta), V_{0}(\eta), W_{0}(\eta)\right)^{T}+\epsilon(\tilde{U}(\eta), \tilde{V}(\eta), \tilde{W}(\eta))^{T} \exp (\mathrm{i} \lambda \phi),
$$

which leads to the (axisymmetric) rotating-disk equations at $O\left(\epsilon^{0}\right)$ and an eigenvalue problem at $O(\epsilon)$; here $\epsilon$ is some small perturbation parameter. The eigenvalue problem for $\lambda$ can be solved numerically by computing the eigenvalues of an appropriately discretized system via a QZ algorithm. Approaching the problem in this way allows one to vary $\hat{W}_{e}$, stepping along the branches to the $O\left(\epsilon^{0}\right)$ rotating-disk problem, to locate any critical values of $\hat{W}_{e}$ for which $\lambda$ is an integer (to satisfy the azimuthal periodicity condition). Computations of this sort suggest that at least one bifurcation point exists, with the non-axisymmetric solution in the neighbourhood of the bifurcation point having an azimuthal wavenumber of two.

Investigation of the bifurcation point using this numerical approach suggests that the bifurcated solution has a rather special form (at least locally): the non-axisymmetry is only introduced into the radial and azimuthal velocity components, and the solution is of the form

$$
\begin{gathered}
\hat{U}(\eta, \phi)=U_{0}+U_{1} \cos (2 \phi), \\
\hat{V}(\eta, \phi)=V_{0}, \\
\hat{W}(\eta, \phi)=W_{0}-U_{1} \sin (2 \phi) .
\end{gathered}
$$

A consequence of this special form is that terms of the form $\mathrm{e}^{4 i \phi}$ are never generated and the solution described by (A 9)-(A 11) is an exact solution of the NavierStokes equations corresponding to the nonlinear, non-axisymmetric flow above a rotating disk. Although (A 5)-(A 7) were derived under a formal boundary-layer approximation, the solution is exact for an azimuthal dependence of the form (A 9)(A 11) since the neglected terms of $O\left(E / r^{2}\right)$ in (A 5)-(A 7) cancel exactly. Furthermore, it is straightforward to show that when $\hat{V}$ is independent of the azimuthal coordinate $\phi$, the $n=2$ solution is the only form for which the series terminates.

We note that this form of solution is similar to that considered previously by Hall, Balakumar \& Papageorgiou (1992), who discussed a class of flows associated with a rotating disk in a fluid with a stagnation point flow at infinity. We shall make further comments on the connection with this work later. 
To describe the bifurcation point we can introduce a perturbation

$$
\hat{W}_{e}=W_{b i f}+\epsilon, \quad|\epsilon| \ll 1,
$$

where $\hat{W}_{e}=W_{b i f}$ is the critical value at which the bifurcation occurs and an expansion of the form (A 9)-(A 11) can be introduced where

$$
\begin{gathered}
U_{0}=U_{0}^{*}+\epsilon U_{1}^{*}+\cdots \\
V_{0}=V_{0}^{*}+\epsilon V_{1}^{*}+\cdots \\
W_{0}=W_{0}^{*}+\epsilon W_{1}^{*}+\cdots \\
U_{1}=\epsilon^{1 / 2} u_{1}+\epsilon^{3 / 2} u_{3}+\cdots .
\end{gathered}
$$

At $O\left(\epsilon^{0}\right)$ we have the rotating disk equations for $\left(U_{0}^{*}, V_{0}^{*}, W_{0}^{*}\right)^{T}$ evaluated at $\hat{W}_{e}=W_{b i f}$; obviously $W_{b i f}$ remains undetermined at this order.

At $O\left(\epsilon^{1 / 2}\right)$ we obtain a linear eigenvalue problem for $u_{1}$ of the form

$$
2 U_{0}^{*} u_{1}+V_{0}^{*} u_{1}^{\prime}=u_{1}^{\prime \prime}
$$

where $u_{1}=0$ at $\eta=0$ and as $\eta \rightarrow-\infty$. We thus determine the critical parameter value (numerically) to be

$$
W_{\text {bif }} \approx-0.14485 \text {, }
$$

in agreement with the approach discussed above. We can therefore write

$$
u_{1}=A \tilde{u},
$$

where $A$ is an amplitude measure that remains undetermined at this order, and $\tilde{u}$ is normalized so that $\tilde{u}^{\prime}(0)=1$.

At $O(\epsilon)$, as noted above, we only have the 'mean flow' terms and the solution can be written as

$$
\left(U_{1}^{*}, V_{1}^{*}, W_{1}^{*}\right)^{T}=\left(U_{1 H}, V_{1 H}, W_{1 H}\right)^{T}+A^{2}\left(\tilde{u}_{2}, \tilde{v}_{2}, \tilde{w}_{2}\right)^{T} .
$$

The terms $\left(U_{1 H}, V_{1 H}, W_{1 H}\right)^{T}$ are the appropriate Taylor series corrections to the rotating disk solution for a perturbation about the bifurcation point,

$$
U_{1 H}=\left.\frac{\partial U_{0}^{*}}{\partial \hat{W}_{e}}\right|_{\hat{W}_{e}=W_{b i f}}, \quad V_{1 H}=\left.\frac{\partial V_{0}^{*}}{\partial \hat{W}_{e}}\right|_{\hat{W}_{e}=W_{b i f}}, \quad W_{1 H}=\left.\frac{\partial W_{0}^{*}}{\partial \hat{W}_{e}}\right|_{\hat{W}_{e}=W_{b i f}} .
$$

The terms $\left(\tilde{u}_{2}, \tilde{v}_{2}, \tilde{w}_{2}\right)^{T}$ satisfy the inhomogeneous, linear system

$$
\begin{gathered}
\mathscr{L}_{0}\left(\tilde{u}_{2}, \tilde{v}_{2}, \tilde{w}_{2}\right)^{T}=\left(\tilde{u}_{1}^{2}, 0,0\right)^{T}, \\
2 \tilde{u}_{2}+\tilde{v}_{2}^{\prime}=0,
\end{gathered}
$$

with boundary conditions $\tilde{u}_{2}=\tilde{v}_{2}=\tilde{w}_{2}=0$ on $\eta=0$ and $\tilde{u}_{2} \rightarrow 0, \tilde{w}_{2} \rightarrow 0$ as $\eta \rightarrow-\infty$. Here $\mathscr{L}_{0}$ represents a linearized operator arising from a perturbation of the axisymmetric form of (A 5) and (A 6).

At $O\left(\epsilon^{3 / 2}\right)$ the governing system reduces to

$$
2 U_{0}^{*} u_{3}+V_{0}^{*} u_{3}^{\prime}-u_{3}^{\prime \prime}=-2\left(A\left[U_{1 H} \tilde{u}_{1}\right]+A^{3}\left[\tilde{u}_{1} \tilde{u}_{2}\right]\right) .
$$

This system obviously requires that an orthogonality condition be satisfied and therefore determines $A$ through the amplitude equation

$$
A\left(A^{2}-C\right)=0 .
$$

The amplitude of the bifurcated non-axisymmetric solution is $A= \pm C^{1 / 2}$, and $A=0$ 
corresponds to the usual axisymmetric rotating disk state. Here $C$ can be determined in terms of an integral involving $\tilde{u}_{1}, \tilde{u}_{2}, U_{1 H}$ and the solution to the adjoint problem. We note that the sign of the constant term $C$ is obviously dependent (through $U_{1 H}$ ) on the sign of the perturbation about $W_{b i f}$; therefore we expect that the bifurcated solution branch will only exist to one side of the critical value $\hat{W}_{e}=W_{b i f}$.

The full nonlinear system governing the unknowns $U_{0}, V_{0}, W_{0}, U_{1}$, as defined by (A 9)-(A 11), is simply

$$
\begin{gathered}
U_{0}^{2}+V_{0} U_{0}^{\prime}-W_{0}^{2}+U_{1}^{2}=U_{0}^{\prime \prime}-\hat{W}_{e}^{2}, \\
2 U_{0} W_{0}+V_{0} W_{0}^{\prime}=W_{0}^{\prime \prime}, \\
2 U_{0}+V_{0}^{\prime}=0, \\
2 U_{0} U_{1}+V_{0} U_{1}^{\prime}=U_{1}^{\prime \prime},
\end{gathered}
$$

with boundary conditions $U_{0}=U_{1}=V_{0}=0, W_{0}=1$ on $\eta=0$ and $U_{0}, U_{1} \rightarrow 0$, $W_{0} \rightarrow \hat{W}_{e}$ as $\eta \rightarrow-\infty$. An investigation of this system has been performed using the bifurcation and continuation package AUTO (Doedel \& Wang 1995), the results of which are shown in figure 18. Figure 18(b) shows the location of the bifurcated solution branch for the non-axisymmetric states relative to the well known rotating disk branches. Figure 18(a) shows the behaviour of an amplitude measure, $U_{1}^{\prime}(0)$, for varying $\hat{W}_{e}$. As noted above there is a square-root-like behaviour at $\hat{W}_{e}=W_{b i f}$; however the $\hat{W}_{e} \ll 1$ region appears to be more complicated. Numerical results suggest the presence of a growing boundary-layer scale as $\hat{W}_{e} \rightarrow 0^{-}$, and we have been unable to continue the non-axisymmetric branch into the region $\hat{W}_{e}>0$.

A likely scenario for the limit $\left|\hat{W}_{e}\right| \ll 1$ involves the development of a double boundary-layer structure. In an inner layer $(\eta=O(1))$ adjacent to the disk the velocity components remain $O(1)$ with an algebraic decay of

$$
\begin{gathered}
U_{0} \sim-3 / \eta^{2}, \\
V_{0} \sim-6 / \eta, \\
W_{0} \sim W_{00} / \eta^{2}, \\
U_{1} \sim \pm\left(9+W_{00}^{2}\right)^{1 / 2} / \eta^{2},
\end{gathered}
$$

as $\eta \rightarrow-\infty$, where $W_{00}$ is a constant. The form of (A 30)-(A 33) together with the edge conditions suggest an outer layer of the form

$$
\begin{gathered}
U_{0}=\left|\hat{W}_{e}\right| \tilde{U}_{0}(\tilde{\eta}), \\
V_{0}=\left|\hat{W}_{e}\right|^{1 / 2} \tilde{V}_{0}(\tilde{\eta}), \\
W_{0}=\left|\hat{W}_{e}\right| \tilde{W}_{0}(\tilde{\eta}), \\
U_{1}=\left|\hat{W}_{e}\right| \tilde{U}_{1}(\tilde{\eta}),
\end{gathered}
$$

with a scaled outer-layer coordinate $\tilde{\eta}=\left|\hat{W}_{e}\right|^{1 / 2} \eta$.

It is possible to make some comparisons between the predicted asymptotic form of solution and numerical results obtained in the limit $\hat{W}_{e} \rightarrow 0^{-}$. In particular, the inner-layer behaviour described by (A 30)-(A 33) suggests that $\eta V(\eta) \rightarrow-6$ as $\eta \rightarrow-\infty$; it is this quantity that is shown in figure 19(a). Similarly, (A 30)-(A 33) show 

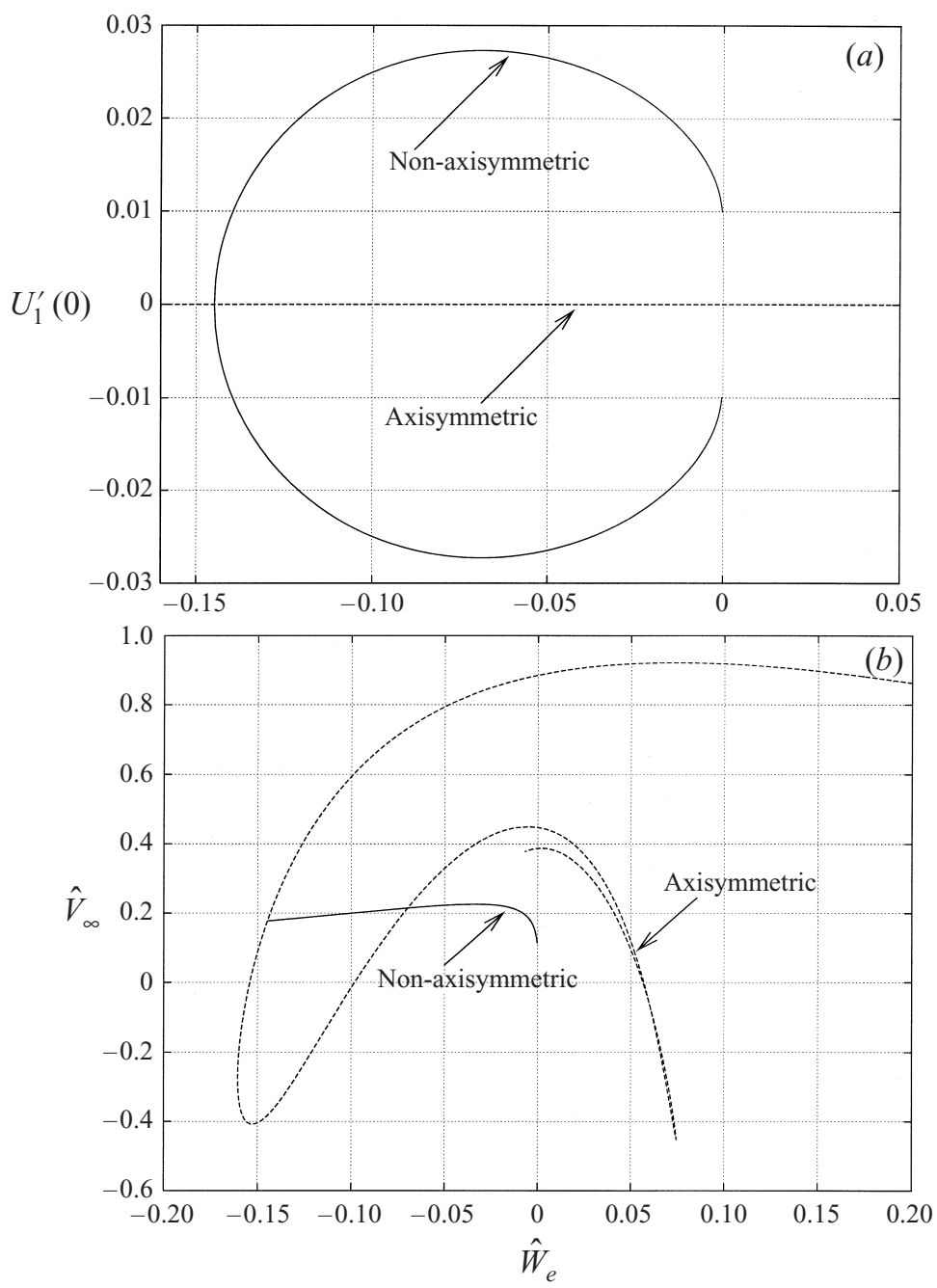

FIgURE 18. (a) A measure of the amplitude of the non-axisymmetric component $U_{1}$. (b) The location of the non-axisymmetric branch in relation to the axisymmetric branches. Here $\hat{V}_{\infty}=V_{0}(\eta \rightarrow-\infty)$.

that

$$
\begin{gathered}
\left(\frac{W_{0}}{U_{0}}\right)^{2} \sim \frac{W_{00}^{2}}{9}, \\
\left\{\left(\frac{U_{1}}{U_{0}}\right)^{2}-1\right\} \sim \frac{W_{00}^{2}}{9},
\end{gathered}
$$

as $\eta \rightarrow-\infty$. The numerical results presented in figure 19 are consistent with these predictions based on the algebraic decay of the inner layer, although we should note that values of $\left|\hat{W}_{e}\right| \sim 10^{-7}$ were necessary to resolve these features.

As we have already noted above, the form of solution (A 9)-(A 11) has been used in a different context by Hall et al. (1992), who considered the unsteady problem for the flow above a rotating disk with a stagnation point flow far from the disk surface. The 

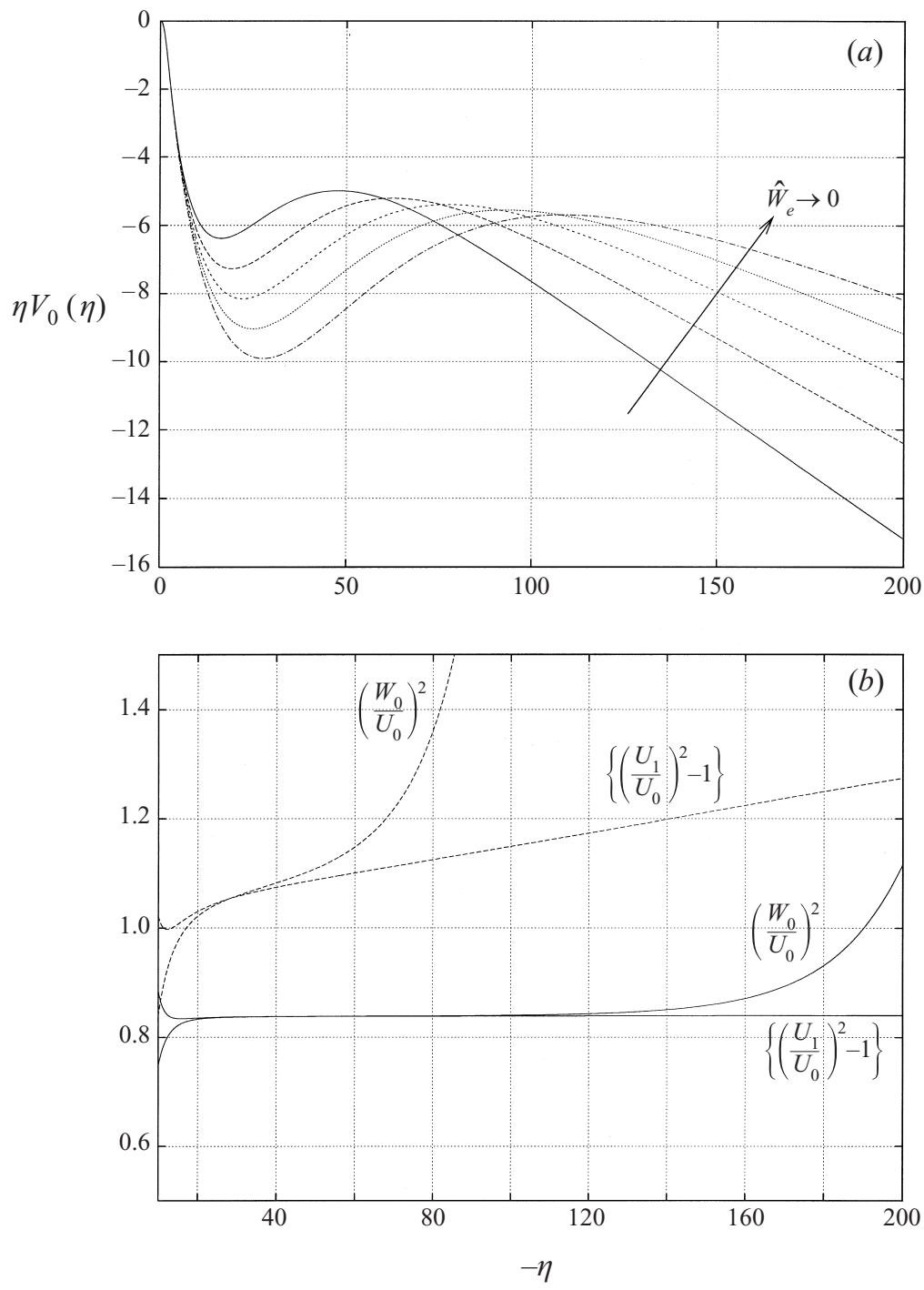

Figure 19. (a) The behaviour of $\eta V_{0}(\eta)$ as $\hat{W}_{e} \rightarrow 0^{-}$. Profiles are shown at $\hat{W}_{e}=10^{-5}, 10^{-6}, \ldots, 10^{-9}$ (b) A comparison of $\left(W_{0} / U_{0}\right)^{2}$ and $\left(U_{1} / U_{0}\right)^{2}-1$ at $\hat{W}_{e}=10^{-6}$ (dashed lines) and $\hat{W}_{e}=10^{-8}$ (solid lines).

relevance of their work to rotating disk flow was in a limiting case, namely that which removed the presence of a stagnation point flow at infinity to leave the far-field fluid stationary. Therefore, the problem was considered to be a nonlinear stability analysis of the flow first considered by von Kármán, that is, the axisymmetric rotating disk state at $\hat{W}_{e}=0$ in our notation. (No non-axisymmetric, steady states were located by Hall et al. in the absence of the stagnation point flow.) The results of Hall et al. showed that a threshold response was obtained leading to a finite-time singularity. It is worth noting however that the description above suggests that $\hat{W}_{e}=0$ is a limiting point for the non-axisymmetric states. It would therefore be of interest to consider the stability of this branch, since if it were linearly stable, a nonlinear perturbation 
beyond the basin of attraction of the axisymmetric rotating disk states may simply lead to these new states when $\hat{W}_{e}<0$ rather than a finite-time breakdown.

\section{REFERENCES}

Benjamin, T. B. 1978 Bifurcation phenomena in steady flows of a viscous fluid. I Theory. Proc. $R$. Soc. Lond. A 359, 1.

Bödewadt, U. T. 1940 Die Drehströmung über festem Grund. Z. Angew. Math. Mech. 20, 241.

BodonyI, R. J. 1973 The laminar boundary layer on a finite rotating disk. PhD dissertation, The Ohio State University.

BodonYI, R. J. 1975 On rotationally symmetric flow above an infinite rotating disk. J. Fluid Mech. 67, 657.

Bodonyi, R. J. 1978 On the unsteady similarity equations for the flow above a rotating disc in a rotating fluid. Q. Appl. Math. Mech. 31, 461.

BodonYI, R. J. \& NG, B. S. 1984 On the stability of the similarity solutions for swirling flow above an infinite rotating disk. J. Fluid Mech. 144, 311.

BODONYI, R. J. \& STEWARTSON, K. 1977 The unsteady laminar boundary layer on a rotating disk in a counter-rotating fluid. J. Fluid Mech. 79, 669.

DiJKstRA, D. 1980 On the relation between adjacent inviscid cell type solutions to the rotating-disk equations. J. Engng Maths 14, 133.

Doedel, E. J. \& WANG, X. 1995 Software for continuation and bifurcation problems in ordinary differential equations. Tech. rep. CRPC-95-2. Center for Research on Parallel Computing, California Institute of Technology.

DuCK, P. W., Foster, M. R. \& HewitT, R. E. $1997 a$ On the boundary layer arising in the spin-up of a stratified fluid in a container with sloping walls. J. Fluid Mech. 335, 233 (referred to herein as DFH).

Hall, P., Balakumar, P. \& Papageorgiou, D. 1992 On a class of unsteady three-dimensional Navier-Stokes solutions relevant to rotating disc flows: threshold amplitudes and finite-time singularities. J. Fluid Mech. 238, 297.

Hewitt, R. E., Davies, P. A., Duck, P. W. \& Foster, M.R. 1999 Spin-up of stratified rotating flows at large Schmidt number: experiment and theory. J. Fluid Mech. (to appear) (referred to herein as HDDF).

Hewitt, R. E., Duck, P. W., Foster, M. R. \& Davies, P. A. $1997 b$ Nonlinear spin-up of a rotating stratified fluid: theory. Trans. ASME J. Fluids Engng 120, 662.

Kármán, T. von 1921 Über laminare und turbulente Reibung. Z. Angew. Math. Phys. 1, 244.

MacCready, P. \& Rhines, P. B. 1991 Buoyant inhibition of Ekman transport on a slope and its effect on stratified spin up. J. Fluid Mech. 223, 631.

OcKeNDON, H. 1972 An asymptotic solution for the steady flow above an infinite rotating disc with suction. Q. J. Mech. Appl. Maths 25, 291.

Rogers, M. H. \& LANCE. G. N. 1960 The rotationally symmetric flow of a viscous fluid in the presence of a rotating disk. J. Fluid Mech. 7, 617.

Stewartson, K., Simpson, C. J. \& Bodonyi, R. J 1982 The unsteady laminar boundary layer on a rotating disk in a counter-rotating fluid: Part 2. J. Fluid Mech. 121, 507.

Thorpe, S. A. 1987 Current and temperature variability on the continental slope. Phil. Trans. R. Soc. Lond. A 323, 471

Zandbergen, P. J. 1979 New solutions of the Kármán problem for rotating flows. Proc. (Methods for Navier-Stokes Problems) Paderborn, Germany, 563.

Zandbergen, P. J. \& Dijkstra, D. 1987 Von Kármán swirling flows. Ann. Rev. Fluid Mech. 19, 465. 